\title{
Synthesis of Enantiopure tert-Butanesulfinamide from tert-Butanesulfinyloxazolidinone
}

\author{
Yong Qin, ${ }^{*}{ }^{\dagger}$ Canhui Wang, ${ }^{\ddagger}$ Zhiyan Huang, ${ }^{\dagger}$ Xue Xiao, ${ }^{\dagger}$ Yaozhong Jiang $* \sharp$ \\ ${ }^{\dagger}$ Department of Chemistry of Medicinal Natural Products, West China School of Pharmacy, \\ Sichuan University, Chengdu 610041, P. R. China \\ ${ }^{\ddagger}$ Chengdu Institute of Organic Chemistry, Graduate School of Chinese Academy of Sciences, \\ Chengdu 610041, P. R. China
}

\section{Contents of Supporting Information}

Page S-1: Title of the paper, author's name and address along with the contents.

Page S-2: Contents of supporting information.

Page S-3: Contents of supporting information.

Page S-4: General methods, general procedure for the preparation of $\mathrm{N}$-alkoxycabonyl-1, 2-diphenylaminoethanols $\mathbf{1}$ and characterization data of compound 1a.

Page S-5: Characterization data of compounds 1b, 1c, 1d, and typical procedure for the preparation of 4,5-diphenyl-1,2,3-oxathiazolidine-2-oxides 2

Page S-6:.Characterization data of compounds endo-2b, exo-2b, endo-2c and exo-2c.

Page S-7: Characterization data of compound endo-2d, exo-2d, and typical procedure for the syntheses of sulfinate $\mathbf{3}$, sulfonamide $\mathbf{4}$ and oxazolidinone $\mathbf{5}$.

Page S-8: Characterization data of compounds $\mathbf{3 b}, \mathbf{4 b}$, and $\mathbf{3 c}$.

Page S-9: Characterization data of compounds $4 \mathbf{c}, \mathbf{3 d}, \mathbf{4 d}$, and $\mathbf{5 .}$ 
Page S-10: Ammonolysis of oxazolidinone 5 and characterization data of compound (R)-6.

Page S-11: Characterization data of compound 7.

Page S-12: ${ }^{1} \mathrm{H}$ NMR spectra of compound 1a.

Page S-13: ${ }^{1} \mathrm{H}$ NMR spectra of compound $\mathbf{1 b}$.

Page S-14: ${ }^{1} \mathrm{H}$ NMR spectra of compound 1c.

Page S-15: ${ }^{1} \mathrm{H}$ NMR spectra of compound $\mathbf{1 d}$.

Page S-16: ${ }^{1} \mathrm{H}$ NMR spectra of compound endo-2b.

Page S-17: ${ }^{13}$ C NMR spectra of compound endo-2b.

Page S-18: ${ }^{1} \mathrm{H}$ NMR spectra of mixture of endo-2b and exo-2b.

Page S-19: ${ }^{1} \mathrm{H}$ NMR spectra of compound endo-2c.

Page S-20: ${ }^{13} \mathrm{C}$ NMR spectra of compound endo-2c.

Page S-21: ${ }^{1} \mathrm{H}$ NMR spectra of mixture of endo-2c and exo-2c.

Page S-22: ${ }^{1} \mathrm{H}$ NMR spectra of compound endo-2d.

Page S-23: ${ }^{13} \mathrm{C}$ NMR spectra of compound endo-2d.

Page S-24: ${ }^{1} \mathrm{H}$ NMR spectra of compound exo-2d.

Page S-25: ${ }^{13}$ C NMR spectra of compound exo-2d.

Page S-26: ${ }^{1} \mathrm{H}$ NMR spectra of compound $\mathbf{3 b}$.

Page S-27: ${ }^{13} \mathrm{C}$ NMR spectra of compound $\mathbf{3 b}$.

Page S-28: ${ }^{1} \mathrm{H}$ NMR spectra of compound 3c.

Page S-29: ${ }^{13}$ C NMR spectra of compound 3c.

Page S-30: ${ }^{1} \mathrm{H}$ NMR spectra of compound 3d. 
Page S-31: ${ }^{13}$ C NMR spectra of compound 3d.

Page S-32: ${ }^{1} \mathrm{H}$ NMR spectra of compound $\mathbf{4 b}$.

Page S-33: ${ }^{13} \mathrm{C}$ NMR spectra of compound $\mathbf{4 b}$.

Page S-34: ${ }^{1}$ H NMR spectra of compound 4c.

Page S-35: ${ }^{13} \mathrm{C}$ NMR spectra of compound $\mathbf{4 c}$.

Page S-36: ${ }^{1} \mathrm{H}$ NMR spectra of compound $\mathbf{4 d}$.

Page S-37: ${ }^{13} \mathrm{C}$ NMR spectra of compound $\mathbf{4 d}$.

Page S-38: ${ }^{1} \mathrm{H}$ NMR spectra of compound 5.

Page S-39: ${ }^{13} \mathrm{C}$ NMR spectra of compound 5.

Page S-40: ${ }^{1} \mathrm{H}$ NMR spectra of (R)-6.

Page S-41: HPLC analysis of crude (R)-6.

Page S-42: HPLC analysis of optical pure (R)-6.

Page S-43: ${ }^{1} \mathrm{H}$ NMR spectra of compound 7.

Page S-44: ${ }^{13} \mathrm{C}$ NMR spectra of compound 7. 


\section{General methods.}

All commercially available reagents were used without further purification. All solvents were dried and distilled before use: THF was distilled from sodium/benzophenone ketyl; dichloromethane were distilled from calcium hydride; toluene was distilled from sodium. Chromatography was conducted by using 200-300-mesh silica gel. IR spectra were recorded on a FT IR spectrometer. NMR spectra were acquired on a $300 \mathrm{MHz}$ NMR spectrometer. HRMS spectra were obtained by the FAB method. HPLC analyses were conducted by using a Diacel Chiralpak AS column.

\section{General procedure for the preparation of $\mathrm{N}$-alkoxycabonyl-1, 2-diphenylaminoethanols}

1.

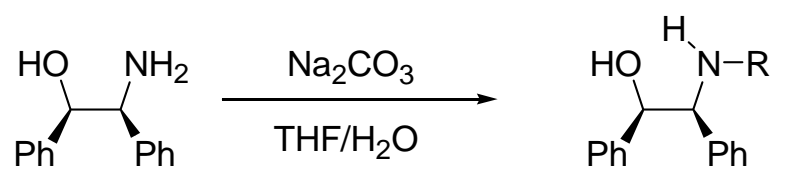

1a $R=T s, 1 b R=B o c$

1c $\mathrm{R}=\mathrm{Cbz}, 1 \mathrm{~d} \mathrm{R}=\mathrm{COOMe}$

To a stirred solution of (1R, 2S)-1,2-diphenylaminoethanol $(2.13 \mathrm{~g}, 10 \mathrm{mmol})$ and $\mathrm{Na}_{2} \mathrm{CO}_{3}$

(2.11g, $20 \mathrm{mmol}$ ) in $120 \mathrm{ml}$ of $\mathrm{THF}$ and $\mathrm{H}_{2} \mathrm{O}(3: 1)$ at $0^{\circ} \mathrm{C}$ was added a solution of $\mathrm{TsCl}$ ( $\mathrm{Boc}_{2} \mathrm{O}$,

$\mathrm{CbzCl}$, or $\mathrm{MeOCOCl}, 11 \mathrm{mmol}$ ) in $5 \mathrm{~mL}$ of THF. After being stirred for $3 \mathrm{~h}$, the mixture was poured into $100 \mathrm{~mL}$ of $\mathrm{H}_{2} \mathrm{O}$ and extracted with dichloromethane to afford crude 1, which was pure enough for next step in most cases. Further purification could be achieved by recrystallization from ethanol.

1a: yield $93 \%, \mathrm{mp} 229-230^{\circ} \mathrm{C} ;[\alpha]^{25}=-97.0$ (c 0.1, THF); IR: 3465, 3324, 1455, 1405, 1313, 1288, 1252, 1154, 1088, $1056 \mathrm{~cm}^{-1} ;{ }^{1} \mathrm{H}$ NMR $\left(\mathrm{CDCl}_{3}\right) \delta 2.33(\mathrm{~s}, 4 \mathrm{H}), 4.55$ (dd, J = 4.4, $7.8 \mathrm{~Hz}$, 
$1 \mathrm{H}), 5.00(\mathrm{~m}, 1 \mathrm{H}), 5.23(\mathrm{~d}, J=7.8 \mathrm{~Hz}, 1 \mathrm{H}), 6.8(\mathrm{~d}, J=7.4 \mathrm{~Hz}, 2 \mathrm{H}), 6.93(\mathrm{~d}, J=7.7 \mathrm{~Hz}, 2 \mathrm{H})$, 7.00-7.26 (m, $8 \mathrm{H}), 7.50$ ( d, $J=8.2 \mathrm{~Hz}, 2 \mathrm{H}$ ) ppm.

1b: yield 97\%, mp 192-193 ${ }^{\circ} \mathrm{C} ;[\alpha]^{25} \mathrm{D}=-57.6\left(\mathrm{c} 1, \mathrm{CHCl}_{3}\right)$; IR: 3378, 2927, 1683, 1523, 1456, 1369, 1293, 1252, $1172 \mathrm{~cm}^{-1} ;{ }^{1} \mathrm{H}$ NMR $\left(\mathrm{CDCl}_{3}\right) \delta 1.26$ (s, $\left.9 \mathrm{H}\right), 2.60$ (s, br, $\left.1 \mathrm{H}\right), 4.97$ (s, br, 1 H), 5.00 (s, br, $1 \mathrm{H}), 5.30(\mathrm{~s}, \mathrm{br}, 1 \mathrm{H}), 7.00(\mathrm{~m}, 4 \mathrm{H}), 7.20(\mathrm{~m}, 6 \mathrm{H}) \mathrm{ppm}$.

1c: yield 94\%, mp 172-173 ${ }^{\circ} \mathrm{C} ;[\alpha]^{25} \mathrm{D}=-67.4\left(\mathrm{c} \mathrm{1}, \mathrm{CHCl}_{3}\right)$; IR: 3348, 3063, 1690, 1540, 1496, 1453, 1441, 1352, 1292, 1258, 1153, 1060, $1034 \mathrm{~cm}^{-1} ;{ }^{1} \mathrm{H}$ NMR $\left(\mathrm{CDCl}_{3}\right) \delta 2.40(\mathrm{~s}, \mathrm{br}, 1 \mathrm{H})$, $5.05(\mathrm{~m}, 4 \mathrm{H}), 5.6(\mathrm{~s}, \mathrm{br}, 1 \mathrm{H})$, 6.90-7.12 (m, $4 \mathrm{H})$, 7.23-7.26 (m, $6 \mathrm{H})$, 7.30-7.65 (m, $5 \mathrm{H})$. 1d: yield 99\%, mp 175-176 ${ }^{\circ} \mathrm{C} ;[\alpha]^{25} \mathrm{D}=-62.1\left(\mathrm{c} \mathrm{1}, \mathrm{CHCl}_{3}\right)$; IR: 3352, 3032, 2944, 1691, 1541, 1498, 1454, 1352, 1309, 1289, 1260, 1216, 1193, 1080, $1060 \mathrm{~cm}^{-1} ;{ }^{1} \mathrm{H} \mathrm{NMR}\left(\mathrm{CDCl}_{3}\right) \delta 2.38(\mathrm{~s}$, br, $1 \mathrm{H}), 3.65(\mathrm{~s}, 3 \mathrm{H}), 5.02(\mathrm{~m}, 1 \mathrm{H}), 5.08(\mathrm{~m}, 1 \mathrm{H}), 5.48(\mathrm{~m}, 1 \mathrm{H}), 7.02(\mathrm{~m}, 4 \mathrm{H}), 7.25(\mathrm{~m}, 6 \mathrm{H})$ ppm.

Typical procedure for the preparation of 4,5-diphenyl-1,2,3-oxathiazolidine-2-oxides 2.

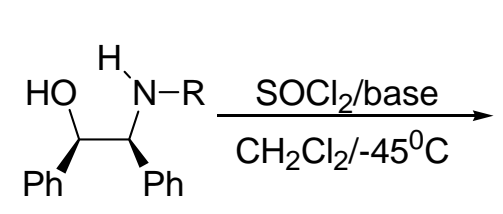

1a $R=T s, 1 b$ R=Boc 1c $\mathrm{R}=\mathrm{Cbz}, 1 \mathrm{~d} \mathrm{R}=\mathrm{COOMe}$<smiles>[2H]N1C(c2ccccc2)C(c2ccccc2)O[Si]1=O</smiles>

endo-2<smiles>O=S1OC(c2ccccc2)C(c2ccccc2)N1P</smiles>

exo-2

Under $\mathrm{N}_{2}$, to a stirred solution of $1(1 \mathrm{mmol})$ and $4-(\mathrm{N}, \mathrm{N}$-dimethylamino)pyridine $(3 \mathrm{mmol})$ in $30 \mathrm{~mL}$ of dried $\mathrm{CH}_{2} \mathrm{Cl}_{2}$ at $-45^{\circ} \mathrm{C}$ was added $\mathrm{SOCl}_{2}(1 \mathrm{mmol})$. After being stirred for $1 \mathrm{~h}$, the mixture was quenched with $\mathrm{H}_{2} \mathrm{O}(5 \mathrm{~mL})$ at $-45^{\circ} \mathrm{C}$ and was allowed to warm to room temperature. The aqueous phase was extracted with $\mathrm{CH}_{2} \mathrm{Cl}_{2}$, the combined organic phases were dried with $\mathrm{MgSO}_{4}$ and evaporated to afford a crude mixture of 2-endo and 2-exo. The 
ratio of endo-2 and exo-2 (table 2) was determined by ${ }^{1} \mathrm{H}$ NMR of crude 2 with integration of proton at position-5. Pure endo-2d and exo-2d were obtained by chromatography (EtOAc/petroleum 1:5). For the inseparable endo and exo mixture of $\mathbf{2 b}$, endo and exo mixture of $\mathbf{2 c}$, purification was achieved by recrystallization (after chromatography, EtOAc/petroleum 1:7) to afford pure endo-2b (EtOAc/petroleum 1:12) and pure endo-2c (EtOAc/petroleum 1:12) respectively.

For endo-2b: $\mathrm{mp} 188-189^{\circ} \mathrm{C} ;[\alpha]^{25} \mathrm{D}=-277.7\left(\mathrm{c} 1, \mathrm{CHCl}_{3}\right)$; IR: 2979, 1727, 1700, 1456, 1369, 1324, 1186, $1140 \mathrm{~cm}^{-1} ;{ }^{1} \mathrm{H}$ NMR $\left(\mathrm{CDCl}_{3}\right) \delta 1.49(\mathrm{~s}, 9 \mathrm{H}), 5.34(\mathrm{~d}, J=6.7 \mathrm{~Hz}, 1 \mathrm{H}), 5.95(\mathrm{~d}, J=$ $6.6 \mathrm{~Hz}, 1 \mathrm{H}), 7.01(\mathrm{~d}, J=6.0 \mathrm{~Hz}, 2 \mathrm{H}), 7.16(\mathrm{~m}, 8 \mathrm{H}) \mathrm{ppm} ;{ }^{13} \mathrm{C} \mathrm{NMR}\left(\mathrm{CDCl}_{3}\right) \delta$ 28.1, 63.7, 84.2, 89.4, 126.8, 126.9, 127.6, 127.9, 127.9, 128.2, 128.5, 133.2, 134.7 ppm; Anal. Calcd for $\mathrm{C}_{19} \mathrm{H}_{21} \mathrm{~N}_{1} \mathrm{O}_{4} \mathrm{~S}_{1}$ : C, 63.49; H, 5.89; N, 3.90; S, 8.92. Found: C, 63.22; H, $5.89 ; \mathrm{N}, 3.94 ; \mathrm{S}, 9.22$. For exo-2b: ${ }^{1} \mathrm{H}$ NMR $\left(\mathrm{CDCl}_{3}\right) \delta 1.30(\mathrm{~s}, 9 \mathrm{H}), 5.34(\mathrm{~d}, J=6.7 \mathrm{~Hz}, 1 \mathrm{H}), 6.57(\mathrm{~s}, \mathrm{br}, 1 \mathrm{H}), 6.80(\mathrm{~d}$, $\mathrm{J}=8.1 \mathrm{~Hz}, 2 \mathrm{H}), 6.94(\mathrm{~d}, \mathrm{~J}=8.0 \mathrm{~Hz}, 2 \mathrm{H}), 7.10(\mathrm{~m}, 6 \mathrm{H}) \mathrm{ppm}$.

For endo-2c: $\mathrm{mp} 127-128^{\circ} \mathrm{C} ;[\alpha]^{25} \mathrm{D}=-242.6\left(\mathrm{c} 1, \mathrm{CHCl}_{3}\right)$; IR: 3030, 1702, 1498, 1456, 1394, 1322, 1186, 1153, 1080, 1033, 956, $939 \mathrm{~cm}^{-1} ;{ }^{1} \mathrm{H} \mathrm{NMR}\left(\mathrm{CDCl}_{3}\right) \delta 5.22$ and $5.30(\mathrm{AB}, J=12 \mathrm{~Hz}$ $2 \mathrm{H}), 5.39(\mathrm{~d}, J=6.6 \mathrm{~Hz}, 1 \mathrm{H}), 5.98(\mathrm{~d}, J=6.7 \mathrm{~Hz}, 1 \mathrm{H}), 6.98(\mathrm{~d}, J=6.6 \mathrm{~Hz}, 1 \mathrm{H}), 7.01(\mathrm{~d}, J=$ $7.8 \mathrm{~Hz}, 2 \mathrm{H}), 71.4(\mathrm{~m}, 9 \mathrm{H}), 7.20(\mathrm{~m}, 4 \mathrm{H}) \mathrm{ppm} ;{ }^{13} \mathrm{C} \mathrm{NMR}\left(\mathrm{CDCl}_{3}\right) \delta$ 63.8, 68.7, 89.6, 126.7, 127.0, 127.8, 128.0, 128.3, 128.5, 128.6, 128.6, 132.9, 134.4, 135.0 ppm; Anal. Calcd for $\mathrm{C}_{22} \mathrm{H}_{19} \mathrm{~N}_{1} \mathrm{O}_{4} \mathrm{~S}_{1}$ : C, 67.16; H, 4.87; N, 3.56; S, 8.15. Found: C, 67.11; H, 4.88; N, 3.60; S, 7.94. For exo-2c: ${ }^{1} \mathrm{H}$ NMR $\left(\mathrm{CDCl}_{3}\right) \delta 5.22$ and $5.30(\mathrm{AB}, J=12 \mathrm{~Hz}, 2 \mathrm{H}), 5.39(\mathrm{~d}, J=6.6 \mathrm{~Hz}, 1 \mathrm{H})$, $6.60(\mathrm{~s}, \mathrm{br}, 1 \mathrm{H}), 6.80(\mathrm{~d}, J=6.6 \mathrm{~Hz}, 2 \mathrm{H}), 7.02(\mathrm{~d}, J=6.6 \mathrm{~Hz}, 2 \mathrm{H}), 7.10(\mathrm{~m}, 7 \mathrm{H}), 7.26(\mathrm{~m}, 4 \mathrm{H})$ ppm. 
For endo-2d: $\mathrm{mp} 143-144^{\circ} \mathrm{C} ;[\alpha]^{25} \mathrm{D}=-299.3\left(\mathrm{C} 1, \mathrm{CHCl}_{3}\right)$; IR: 2958, 1712, 1496, 1437, 1328, 1277, 1187, 1145, $1084 \mathrm{~cm}^{-1} ;{ }^{1} \mathrm{H}$ NMR $\left(\mathrm{CDCl}_{3}\right) \delta 3.83(\mathrm{~s}, 3 \mathrm{H}), 5.34(\mathrm{~d}, \mathrm{~J}=6.7 \mathrm{~Hz}, 1 \mathrm{H}), 5.95(\mathrm{~d}$, $J=6.7 \mathrm{~Hz}, 1 \mathrm{H}), 6.99(\mathrm{~d}, J=6.0 \mathrm{~Hz}, 2 \mathrm{H}), 7.12(\mathrm{~m}, 8 \mathrm{H}) \mathrm{ppm} ;{ }^{13} \mathrm{C} \mathrm{NMR}\left(\mathrm{CDCl}_{3}\right) \delta 54.1,63.8$, 89.5, 126.7, 127.8, 128.0, 128.0, 128.3, 128.7, 132.9, 134.2 ppm; Anal. Calcd for $\mathrm{C}_{16} \mathrm{H}_{15} \mathrm{~N}_{1} \mathrm{O}_{4} \mathrm{~S}_{1}: \mathrm{C}, 60.55 ; \mathrm{H}, 4.76 ; \mathrm{N}, 4.41 ; \mathrm{S}, 10.10$. Found: $\mathrm{C}, 60.65 ; \mathrm{H}, 4.80 ; \mathrm{N}, 4.44 ; \mathrm{S}$, 10.32 .

For exo-2d: $\mathrm{mp} 165-166^{\circ} \mathrm{C} ;[\alpha]^{25} \mathrm{D}=-104.6\left(\mathrm{c} \mathrm{0.78}, \mathrm{CHCl}_{3}\right)$; IR: 2953, 1746, 1495, 1453, 1440, 1326, 1196, 1135, 1063, 1028, 993, 953, $925 \mathrm{~cm}^{-1} ;{ }^{1} \mathrm{H}$ NMR $\left(\mathrm{CDCl}_{3}\right) \delta 3.81$ (s, br, $\left.3 \mathrm{H}\right) ; 5.35$ (s, br, $1 \mathrm{H}), 6.56(\mathrm{~d}, J=6.6 \mathrm{~Hz}, 1 \mathrm{H}), 6.77(\mathrm{~s}, J=6.3 \mathrm{~Hz}, 2 \mathrm{H}), 6.90(\mathrm{~d}, J=7.0 \mathrm{~Hz}, 2 \mathrm{H}), 7.10(\mathrm{~m}, 6$ H) ppm; ${ }^{13} \mathrm{C} \mathrm{NMR}\left(\mathrm{CDCl}_{3}\right) \delta 53.9,64.8,89.5,126.7,128.0,128.1,128.2,128.3,128.7,128.8$, 131.9, 133.9 ppm; Anal. Calcd for $\mathrm{C}_{16} \mathrm{H}_{15} \mathrm{~N}_{1} \mathrm{O}_{4} \mathrm{~S}_{1}$ : C, 60.55; H, 4.76; N, 4.41; S, 10.10. Found: C, 60.71; H, 4.82; N, 4.48; S, 10.34 .

Typical procedure for the syntheses of sulfinate 3, sulfonamide 4 and oxazolidinone 5.

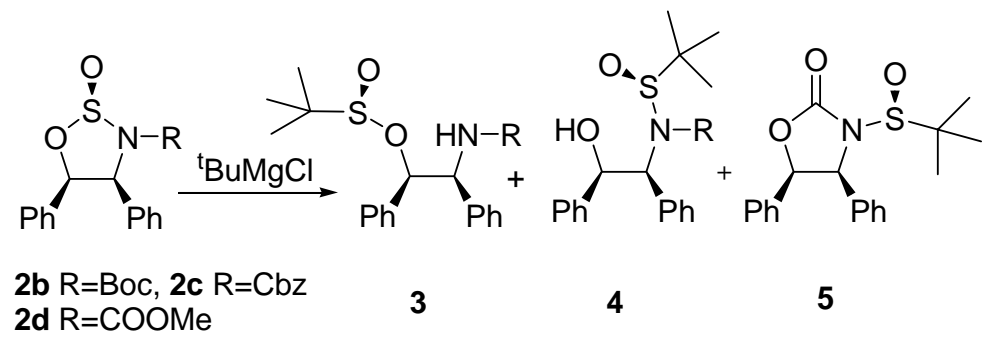

Under $\mathrm{N}_{2}$, to a stirred solution of 4, 5-Diphenyl-1,2,3-oxathiazolidine-2-oxides 2 (endo isomer, $1 \mathrm{mmol})$ in $10 \mathrm{~mL}$ of dry THF at $-45^{\circ} \mathrm{C}$ was added tert-butylmagnesium chloride $(1.5 \mathrm{mmol}$, $0.75 \mathrm{~mL}$ of $2 \mathrm{M}$ solution in $\mathrm{THF})$. After addition, the reaction was stirred at $-45^{\circ} \mathrm{C}$ for $2-4 \mathrm{~h}$ monitored by TLC analysis. Once the starting material was consumed completely, the reaction was quenched with saturated $\mathrm{NH}_{4} \mathrm{Cl}$ aqueous solution $(1 \mathrm{~mL})$ and allowed to warm to room 
temperature. The mixture was diluted with $30 \mathrm{~mL}$ of ethyl acetate and washed with brine, and dried with $\mathrm{MgSO}_{4}$. Concentration and chromatography gave sulfinate $\mathbf{3}$, sulfonamide 4 and oxazolidinone 5 as depicted in table 3.

For sulfinate $3 \mathbf{b}$ : $\mathrm{mp} 195-196{ }^{\circ} \mathrm{C} ;[\alpha]^{25} \mathrm{D}=-114.9\left(\mathrm{c} 0.5, \mathrm{CHCl}_{3}\right)$; IR: $3749,3423,1694,1513$, $1457,1390,1365,1294,1245,1169,1125 \mathrm{~cm}^{-1} ;{ }^{1} \mathrm{H}$ NMR $\left(\mathrm{CDCl}_{3}\right) \delta 1.20(\mathrm{~s}, 9 \mathrm{H}), 1.43(\mathrm{~s}, 9 \mathrm{H})$, $4.96(\mathrm{~d}, \mathrm{~J}=7.8 \mathrm{~Hz}, 1 \mathrm{H}), 5.54(\mathrm{~s}, \mathrm{br}, 1 \mathrm{H}), 6.10(\mathrm{~m}, 1 \mathrm{H}), 6.92(\mathrm{~m}, 4 \mathrm{H}), 7.25(\mathrm{~m}, 6 \mathrm{H}) \mathrm{ppm} ;{ }^{13} \mathrm{C}$ $\operatorname{NMR}\left(\mathrm{CDCl}_{3}\right) \delta 21.8,28.4,58.1,59.8,79.7,84.6,126.9,127.4,127.5,128.0,128.2,128.7$, 138.0, 139.0, 155.1 ppm. HRMS-FAB calcd for $\mathrm{C}_{23} \mathrm{H}_{31} \mathrm{~N}_{1} \mathrm{O}_{4} \mathrm{~S}_{1}(\mathrm{M}+\mathrm{Na}) 440.1873$, found 440.1879.

For sulfinamide $4 \mathrm{~b}: \mathrm{mp} 186-187^{\circ} \mathrm{C} ;[\alpha]^{25} \mathrm{D}=+77\left(\mathrm{c} 0.5, \mathrm{CHCl}_{3}\right)$; IR: $3297,2977,1734,1457$, 1392, 1367, 1280, 1255, 1162, 1064, $977 \mathrm{~cm}^{-1} ;{ }^{1} \mathrm{H}$ NMR $\left(\mathrm{CDCl}_{3}\right) \delta 1.1(\mathrm{~s}, 9 \mathrm{H}), 1.35(\mathrm{~s}, 9 \mathrm{H})$, $3.70(\mathrm{~s}, 1 \mathrm{H}), 4.72(\mathrm{dd}, J=2.7,6.6 \mathrm{~Hz}, 1 \mathrm{H}), 5.82(\mathrm{~d}, J=6.6 \mathrm{~Hz}, 1 \mathrm{H}), 7.28(\mathrm{~m}, 10 \mathrm{H}) \mathrm{ppm} ;{ }^{13} \mathrm{C}$ $\operatorname{NMR}\left(\mathrm{CDCl}_{3}\right) \delta 22.5,27.6,55.7,62.1,80.7,82.6,127.5,128.0,128.2,128.5,128.8,129.4$ 136.0, 136.4, 152.8 ppm; HRMS-FAB calcd for $\mathrm{C}_{23} \mathrm{H}_{31} \mathrm{~N}_{1} \mathrm{O}_{4} \mathrm{~S}_{1}(\mathrm{M}+\mathrm{Na})$ 440.1873, found 440.1871.

For sulfinate 3c: $\mathrm{mp} 153-154{ }^{\circ} \mathrm{C} ;[\alpha]^{25} \mathrm{D}=-112.8\left(\mathrm{c} 1, \mathrm{CHCl}_{3}\right)$; IR: 3401, 2926, 1701, 1519, 1288, 1243, 1219, 1124, $978 \mathrm{~cm}^{-1} ;{ }^{1} \mathrm{H}$ NMR $\left(\mathrm{CDCl}_{3}\right) \delta 1.20(\mathrm{~s}, 9 \mathrm{H}), 5.06(\mathrm{AB}, J=12 \mathrm{~Hz}, 2 \mathrm{H})$, $5.10(\mathrm{~d}, J=3.6 \mathrm{~Hz}, 1 \mathrm{H}), 5.6(\mathrm{~s}, 1 \mathrm{H}), 6.50(\mathrm{~d}, J=7.5 \mathrm{~Hz}, 1 \mathrm{H}), 6.95(\mathrm{~m}, 4 \mathrm{H}), 7.10-7.35(\mathrm{~m}, 11$ H) $\mathrm{ppm} ;{ }^{13} \mathrm{C}$ NMR 21.6, 58.1, 60.1, 66.8, 84.7, 126.7, 127.5, 127.6, 127.9, 128.0, 128.3, 128.4, 128.7, 135.8, 136.6, 136.7, 155.7 ppm; Anal. Calcd for $\mathrm{C}_{26} \mathrm{H}_{29} \mathrm{~N}_{1} \mathrm{O}_{4} \mathrm{~S}_{1}$ : C, 69.15; H, 6.47; N, 3.10; S, 7.10. Found: C, 68.86; H, 6.53; N, 3.14; S, 6.85 . 
For sulfinamide $4 \mathrm{c}: \mathrm{mp} 168-169{ }^{\circ} \mathrm{C} ;[\alpha]^{25} \mathrm{D}=+65.4\left(\mathrm{c} \mathrm{0.5}, \mathrm{CHCl}_{3}\right)$; IR: 3297, 2958, 2926, 1739 , 1456, 1386, 1365, 1269, $1064 \mathrm{~cm}^{-1} ;{ }^{1} \mathrm{H}$ NMR $\left(\mathrm{CDCl}_{3}\right) \delta 1.08(\mathrm{~s}, 9 \mathrm{H}), 3.61(\mathrm{~s}, 1 \mathrm{H}), 4.74(\mathrm{dd}, J$ $=2.1,6.6 \mathrm{~Hz}, 1 \mathrm{H}), 5.06(\mathrm{AB}, J=12 \mathrm{~Hz}, 2 \mathrm{H}), 5.85(\mathrm{~d}, J=6.6 \mathrm{~Hz}, 1 \mathrm{H}), 7.22(\mathrm{~m}, 15 \mathrm{H}) \mathrm{ppm} ;{ }^{13} \mathrm{C}$ $\operatorname{NMR}\left(\mathrm{CDCl}_{3}\right) \delta 22.4,55.7,62.0,69.8,82.1,127.4,128.1,128.3,128.5,128.6,129.0,129.2$ 135.0, 135.5, 136.2 ppm; HRMS-FAB calcd for $\mathrm{C}_{26} \mathrm{H}_{29} \mathrm{~N}_{1} \mathrm{O}_{4} \mathrm{~S}_{1}(\mathrm{M}+\mathrm{Na})$ 474.1725, found 474.1715.

For sulfinate $3 \mathbf{d}$ : $\mathrm{mp} 182-183^{\circ} \mathrm{C} ;[\alpha]^{25} \mathrm{D}=-135.1\left(\mathrm{c} 0.6, \mathrm{CHCl}_{3}\right)$; IR: 3358, 2955, 1729, 1702 , 1530, 1456, 1290, 1251, 1101, $981 \mathrm{~cm}^{-1} ;{ }^{1} \mathrm{H} \mathrm{NMR}\left(\mathrm{CDCl}_{3}\right) \delta 1.21(\mathrm{~s}, 9 \mathrm{H}), 3.67$ (s, $\left.3 \mathrm{H}\right), 4.99$ (dd, $J=1.6,6.6 \mathrm{~Hz}, 1 \mathrm{H}), 5.56(\mathrm{~d}, J=1.8 \mathrm{~Hz}, 1 \mathrm{H}), 6.50(\mathrm{~d}, J=7.0 \mathrm{~Hz}, 1 \mathrm{H}), 6.9(\mathrm{~m}, 4 \mathrm{H}), 7.20$ $(\mathrm{m}, 6 \mathrm{H}) \mathrm{ppm} ;{ }^{13} \mathrm{C} \mathrm{NMR}\left(\mathrm{CDCl}_{3}\right) \delta 21.8,52.3,58.2,60.0,85.2,126.6,127.5,127.6,128.0$, 128.3, 128.7, 135.8, 136.8 ppm; Anal. Calcd for $\mathrm{C}_{20} \mathrm{H}_{25} \mathrm{~N}_{1} \mathrm{O}_{4} \mathrm{~S}_{1}$ : C, 63.97; $\mathrm{H}, 6.71 ; \mathrm{N}, 3.73 ; \mathrm{S}$, 8.54. Found: C, 63.84; H, 6.64; N, 3.80; S, 8.66.

For sulfinamide $4 \mathrm{~d}: \mathrm{mp} 161-162{ }^{\circ} \mathrm{C} ;[\alpha]^{25} \mathrm{D}=+79.6\left(\mathrm{C} 1, \mathrm{CHCl}_{3}\right)$; IR: 3298, 2925, 2854, 1744, 1444, 1272, 1063, $979 \mathrm{~cm}^{-1} ;{ }^{1} \mathrm{H}$ NMR $\left(\mathrm{CDCl}_{3}\right) \delta 1.11(\mathrm{~s}, 9 \mathrm{H}), 3.59(\mathrm{~s}, 1 \mathrm{H}), 3.68(\mathrm{~s}, 3 \mathrm{H}), 4.77$ (dd, $J=2.7,6.6 \mathrm{~Hz}, 1 \mathrm{H}), 5.83(\mathrm{~d}, J=6.3 \mathrm{~Hz}, 1 \mathrm{H}), 7.20-7.34(\mathrm{~m}, 10 \mathrm{H}) \mathrm{ppm} ;{ }^{13} \mathrm{C} \mathrm{NMR}\left(\mathrm{CDCl}_{3}\right)$ $\delta 22.4,55.0,55.7,61.9,77.0,82.1,127.4,128.2,128.3,128.6,129.0,129.1,135.5,136.3$, 154.9 ppm; HRMS-FAB calcd for $\mathrm{C}_{20} \mathrm{H}_{25} \mathrm{~N}_{1} \mathrm{O}_{4} \mathrm{~S}_{1}(\mathrm{M}+\mathrm{Na}) 398.1395$, found 398.1402.

For oxazolidinone 5: $\mathrm{mp} 181-182{ }^{\circ} \mathrm{C} ;[\alpha]^{25} \mathrm{D}=51.5\left(\mathrm{c} 1, \mathrm{CHCl}_{3}\right)$; IR: 1746, 1454, 1338, 1289 , 1216, 1184, 1130, 1101, $1013 \mathrm{~cm}^{-1} ;{ }^{1} \mathrm{H}$ NMR $\left(\mathrm{CDCl}_{3}\right) \delta 1.38(\mathrm{~s}, 9 \mathrm{H}), 5.22(\mathrm{~d}, J=6.6 \mathrm{~Hz}, 1 \mathrm{H})$, $5.95(\mathrm{~d}, J=6.6 \mathrm{~Hz}, 1 \mathrm{H}), 6.92(\mathrm{~m}, 4 \mathrm{H}), 7.11(\mathrm{~m}, 6 \mathrm{H}) \mathrm{ppm} ;{ }^{13} \mathrm{C} \mathrm{NMR}\left(\mathrm{CDCl}_{3}\right) \delta 22.3,61.1,64.1$, 82.3, 126.2, 126.5, 128.0, 128.1, 128.4, 132.4, 134.8, 156.2 ppm; Anal. Calcd for 
$\mathrm{C}_{19} \mathrm{H}_{21} \mathrm{~N}_{1} \mathrm{O}_{3} \mathrm{~S}_{1}: \mathrm{C}, 66.45 ; \mathrm{H}, 6.16 ; \mathrm{N}, 4.08 ; \mathrm{S}, 13.98$. Found: $\mathrm{C}, 64.35 ; \mathrm{H}, 6.36 ; \mathrm{N}, 4.28 ; \mathrm{S}, 14.11$.

HRMS-FAB calcd for $\mathrm{C}_{19} \mathrm{H}_{21} \mathrm{~N}_{1} \mathrm{O}_{3} \mathrm{~S}_{1}(\mathrm{M}+\mathrm{Na}) 366.1134$, found 366.1140 .

\section{Ammonolysis of oxazolidinone 5.}

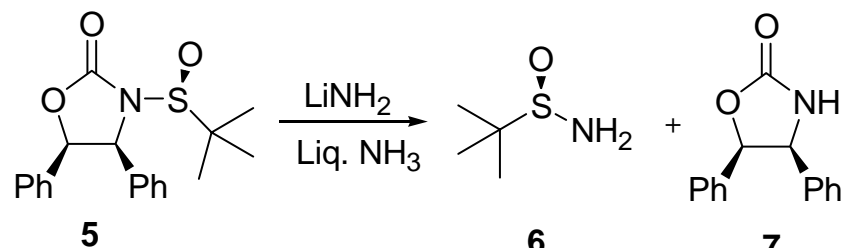

To a solution of liquid ammonia $(20 \mathrm{~mL})$ was added a few crystals of $\mathrm{Fe}\left(\mathrm{NO}_{3}\right)_{3} \cdot 9 \mathrm{H}_{2} \mathrm{O}$, followed by addition of lithium pieces $(0.184 \mathrm{~g}, 8 \mathrm{mmol})$ in a controlled manner while keeping the internal temperature at around $-45^{\circ} \mathrm{C}$. When all lithium was added and the solution became gray, the mixture was stirred for additional $1 \mathrm{~h}$. The mixture was cooled to $-78^{\circ} \mathrm{C}$ and was added a solution of $5(0.687 \mathrm{~g}, 2 \mathrm{mmol})$ in $5 \mathrm{~mL}$ of THF. After being stirred for $0.5 \mathrm{~h}$, the mixture was added solid $\mathrm{NH}_{4} \mathrm{Cl}$ in small portion. The mixture was warmed to room temperature and the ammonia gas escaped from the reaction was trapped by water. The residue was added $2 \mathrm{~mL}$ of $\mathrm{H}_{2} \mathrm{O}$ and $20 \mathrm{~mL}$ of ethyl acetate, and stirred vigorously. The organic phase was separated and the aqueous phase was washed with EtOAc $(2 \times 10 \mathrm{~mL})$. The combined organic phases were washed with brine and dried with $\mathrm{MgSO}_{4}$ and evaporated to dryness. The residue was subjected to chromatography (EtOAc) to afford $7(0.464 \mathrm{~g}, 91 \%)$ and $(\mathrm{R})-6(0.216 \mathrm{~g}, 89 \%)$ with 98.6\% ee. A single recrystallization from hexane afforded (R)-6 with $>99 \%$ ee. The absolute stereochemistry and enantiomeric excess of (R)-6 was established by Chiral HPLC analysis (Diacel Chiralpak AS column, hexane/ethanol 95:5; $1 \mathrm{~mL} / \mathrm{min}, 222 \mathrm{~nm},(\mathrm{R})-\mathbf{6}, \mathrm{r}_{\mathrm{t}}=9.0 \mathrm{~min}$; (S)-6, $\left.\mathrm{r}_{\mathrm{t}}=13.2 \mathrm{~min}\right) . \mathrm{mp} 101-102{ }^{\circ} \mathrm{C} ;{ }^{1} \mathrm{H}$ NMR $\left(\mathrm{CDCl}_{3}\right) \delta 1.18(\mathrm{~s}, 9 \mathrm{H}), 3.82(\mathrm{~s}, \mathrm{br}, 2 \mathrm{H}) \mathrm{ppm}$. 
For compound 7, $\mathrm{mp} 221-222^{\circ} \mathrm{C} ;[\alpha]^{25} \mathrm{D}=-79.2\left(\mathrm{c} 1, \mathrm{CHCl}_{3}\right)$; IR: 3277, 2924, 1748, 1712, 1455, 1426, 1352, 1235, 1094, $1072 \mathrm{~cm}^{-1} ;{ }^{1} \mathrm{H}$ NMR $\left(\mathrm{CDCl}_{3}\right) \delta 5.20(\mathrm{~d}, J=8.1 \mathrm{~Hz}, 1 \mathrm{H}), 5.39(\mathrm{~s}, \mathrm{br}, 1$ H), $5.97(\mathrm{~d}, J=8.2 \mathrm{~Hz}, 1 \mathrm{H}), 6.97(\mathrm{~m}, 4 \mathrm{H}), 7.10(\mathrm{~m}, 6 \mathrm{H}) \mathrm{ppm} ;{ }^{13} \mathrm{C} \mathrm{NMR}\left(\mathrm{CDCl}_{3}\right) \delta$ 61.4, 82.3, $126.1,126.9,127.9,128.1,128.3,134.3,136.0,159.5$ ppm; Anal. Calcd for $\mathrm{C}_{15} \mathrm{H}_{13} \mathrm{~N}_{1} \mathrm{O}_{3}$ : C, 75.30; H, 5.48; N, 5.85. Found: C, 75.17; H, 5.50; N, 5.91. 


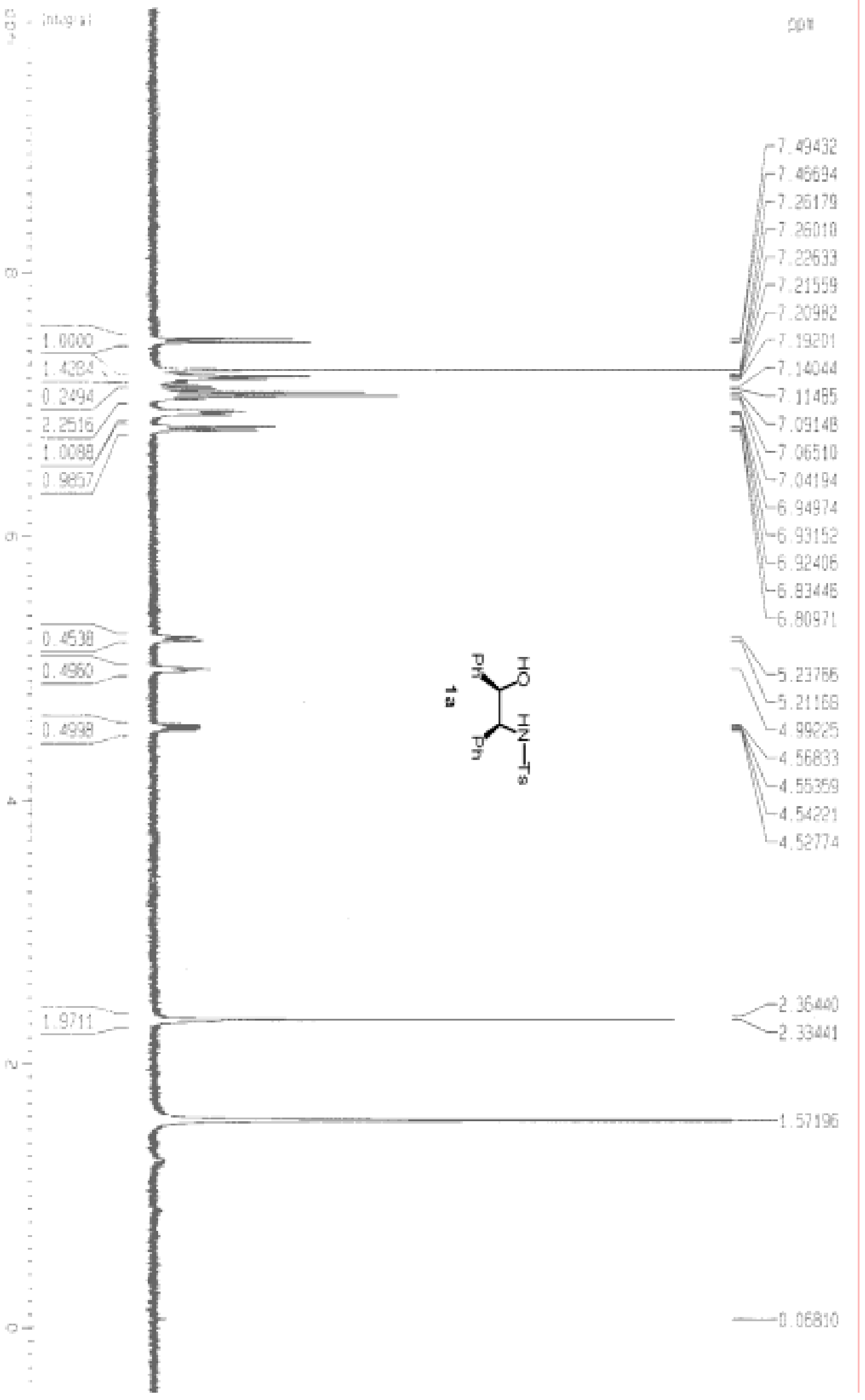



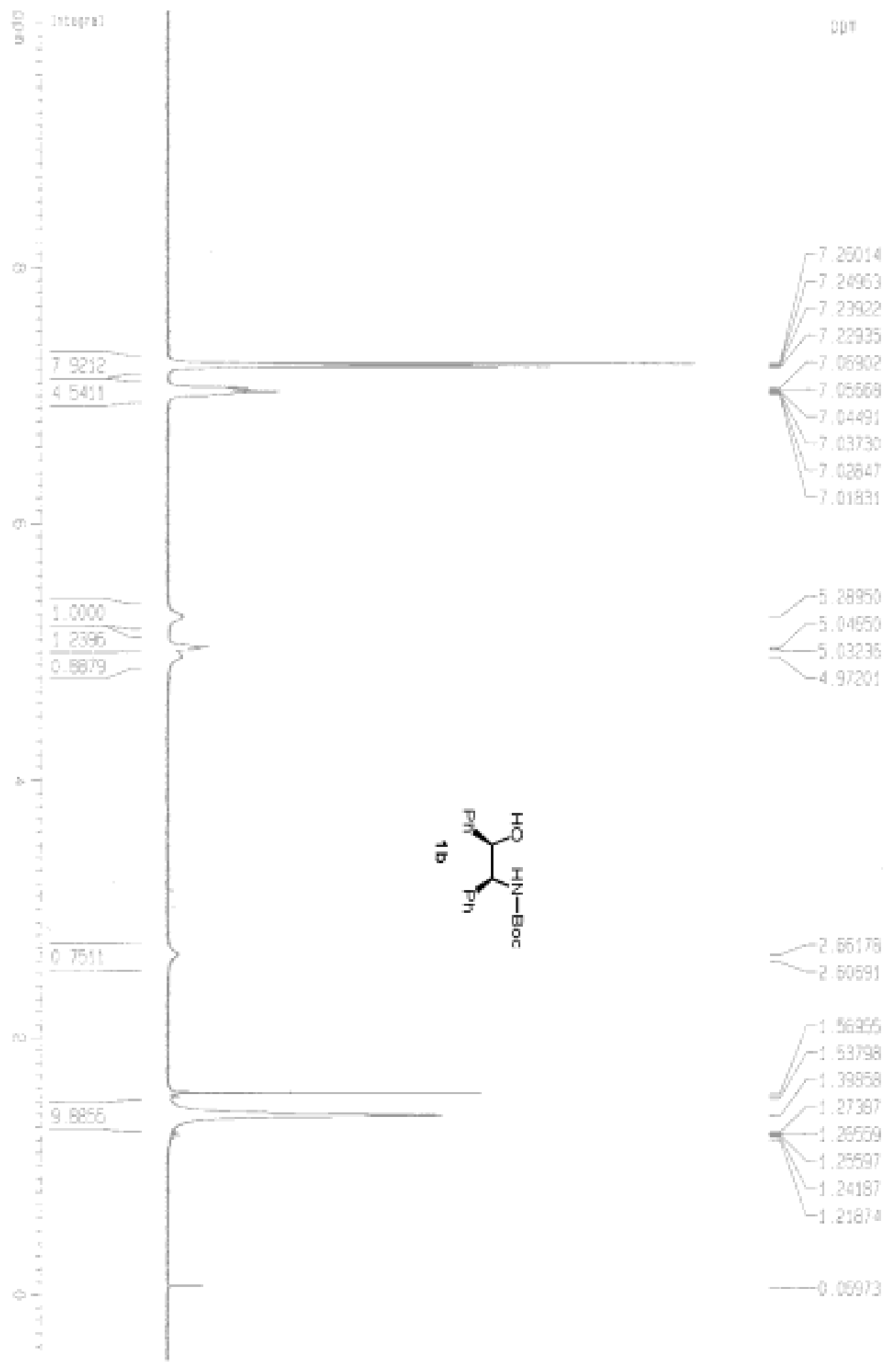

$-720063$

$\sqrt{-7.23922}$

$\sqrt{-7.22935}$

$-7.05900$

$-3.05669$

?

$L_{3} .01839$

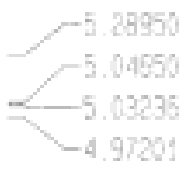

$-2.65175$

-2 50591

$-1.56 y^{4}$

$-1.53793$

$-1.33958$

$-1.27367$

- 1.25559

-1.2559 ?

-1.24187

$-1.29878$

0.05973 


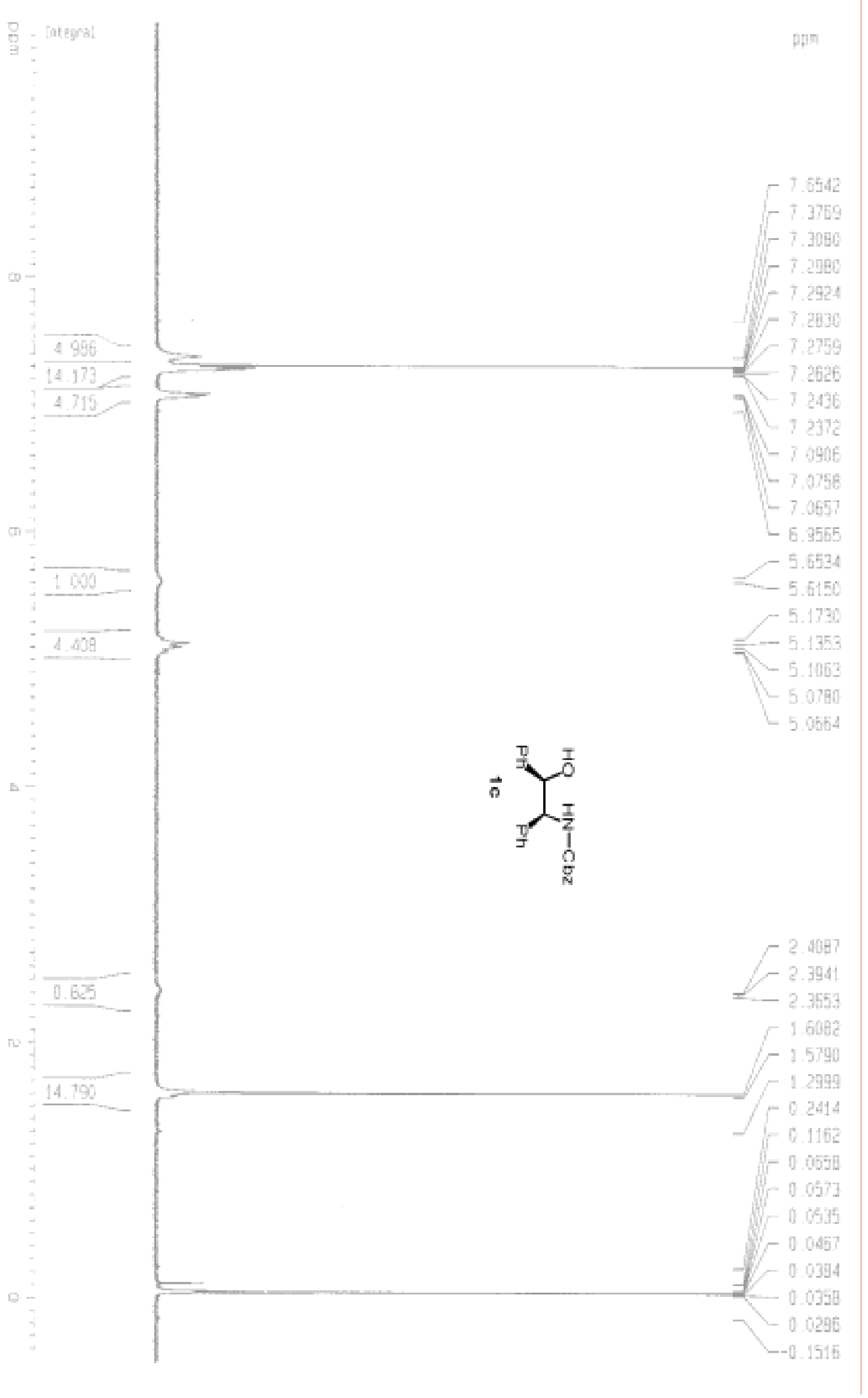



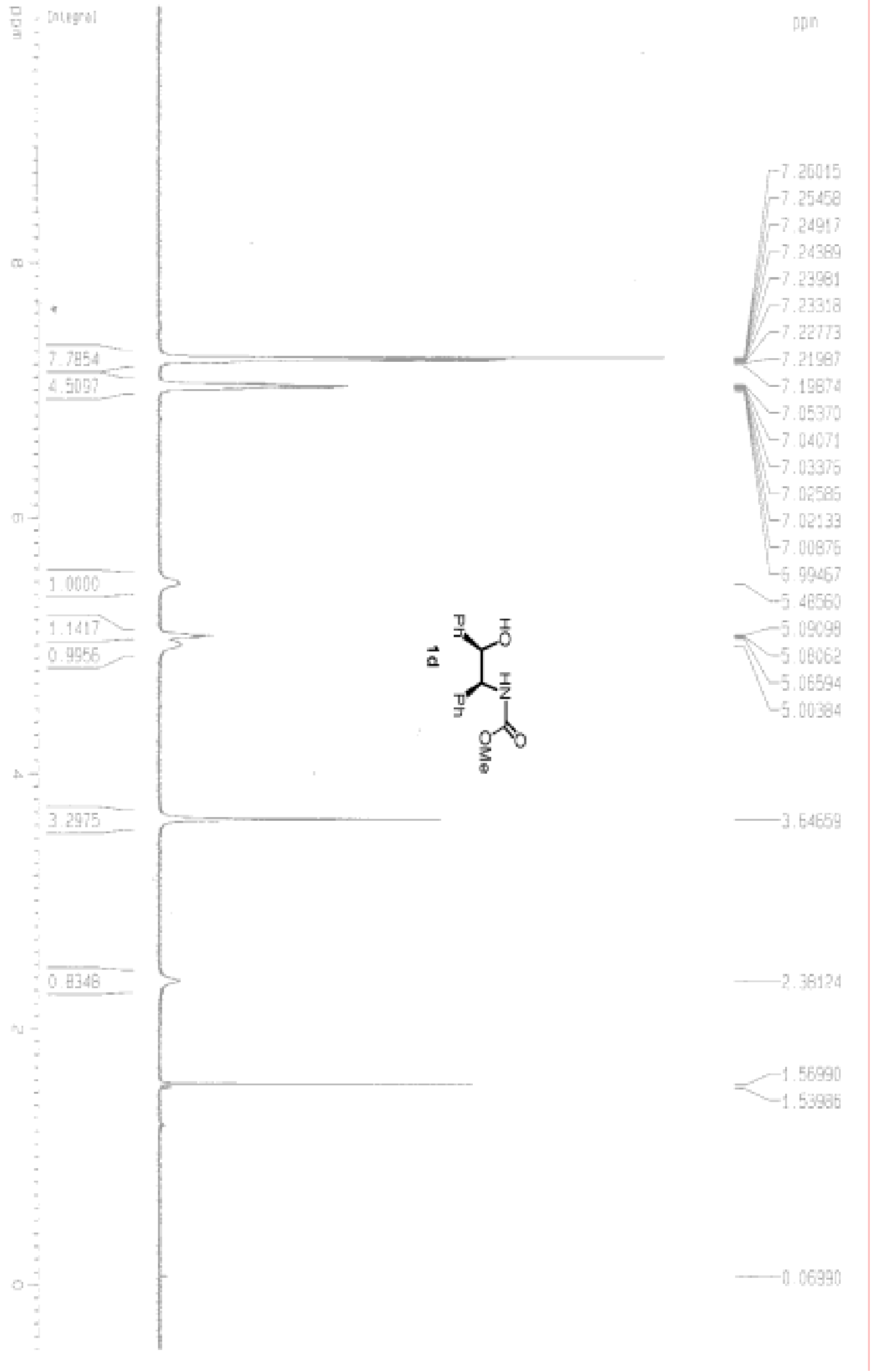


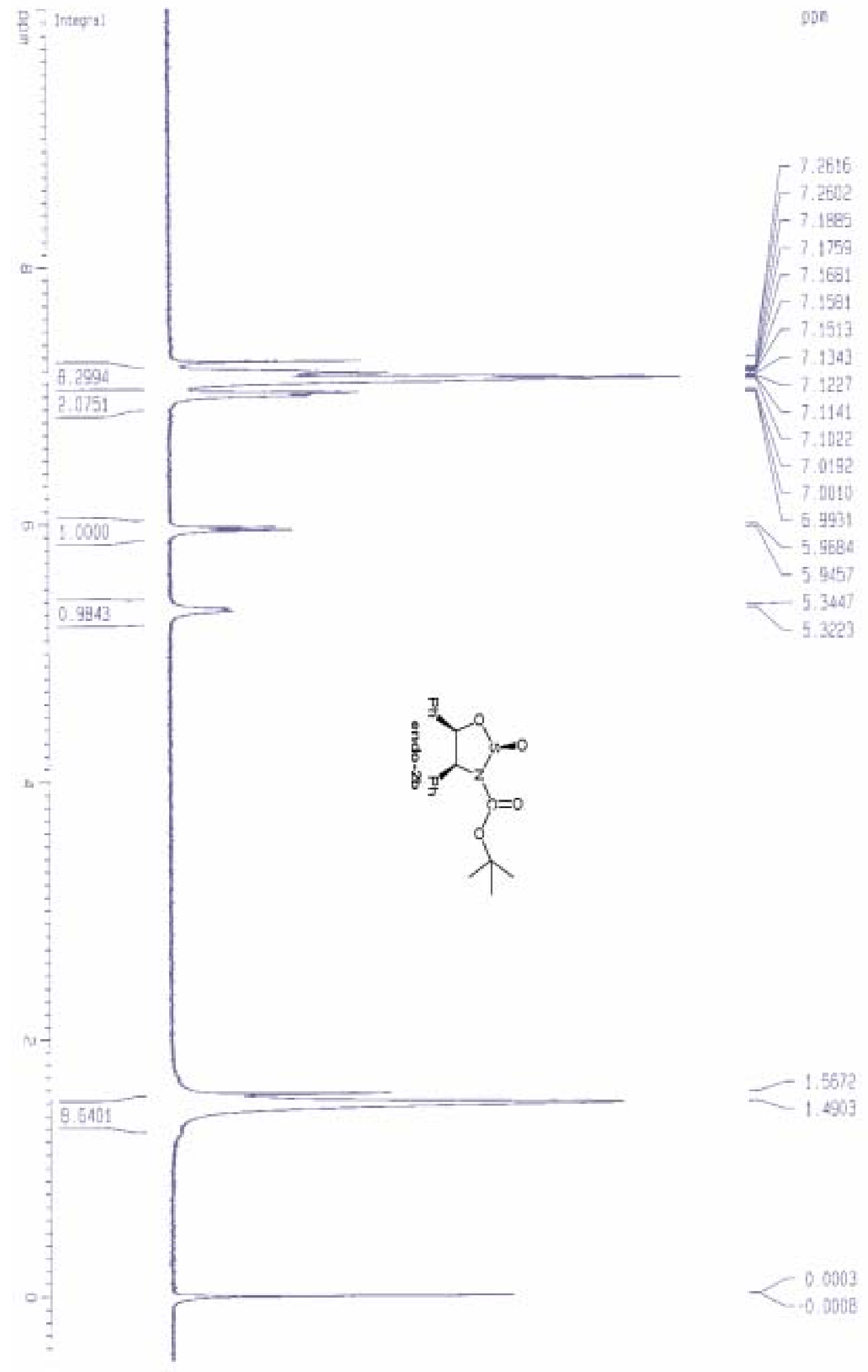



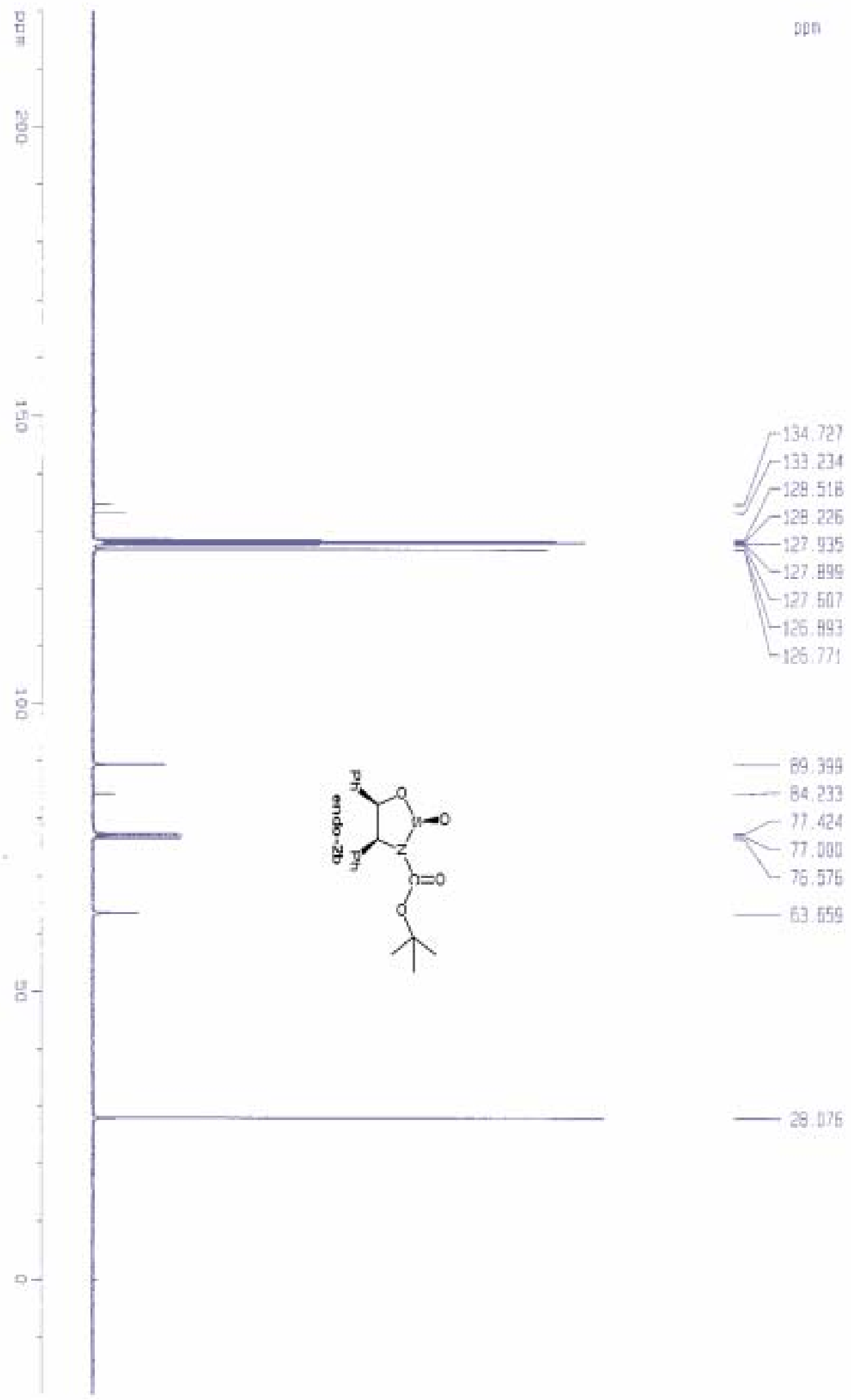

- 69.399 84, 233 77.424

$3-77.000$ $-75.576$ 63.555 


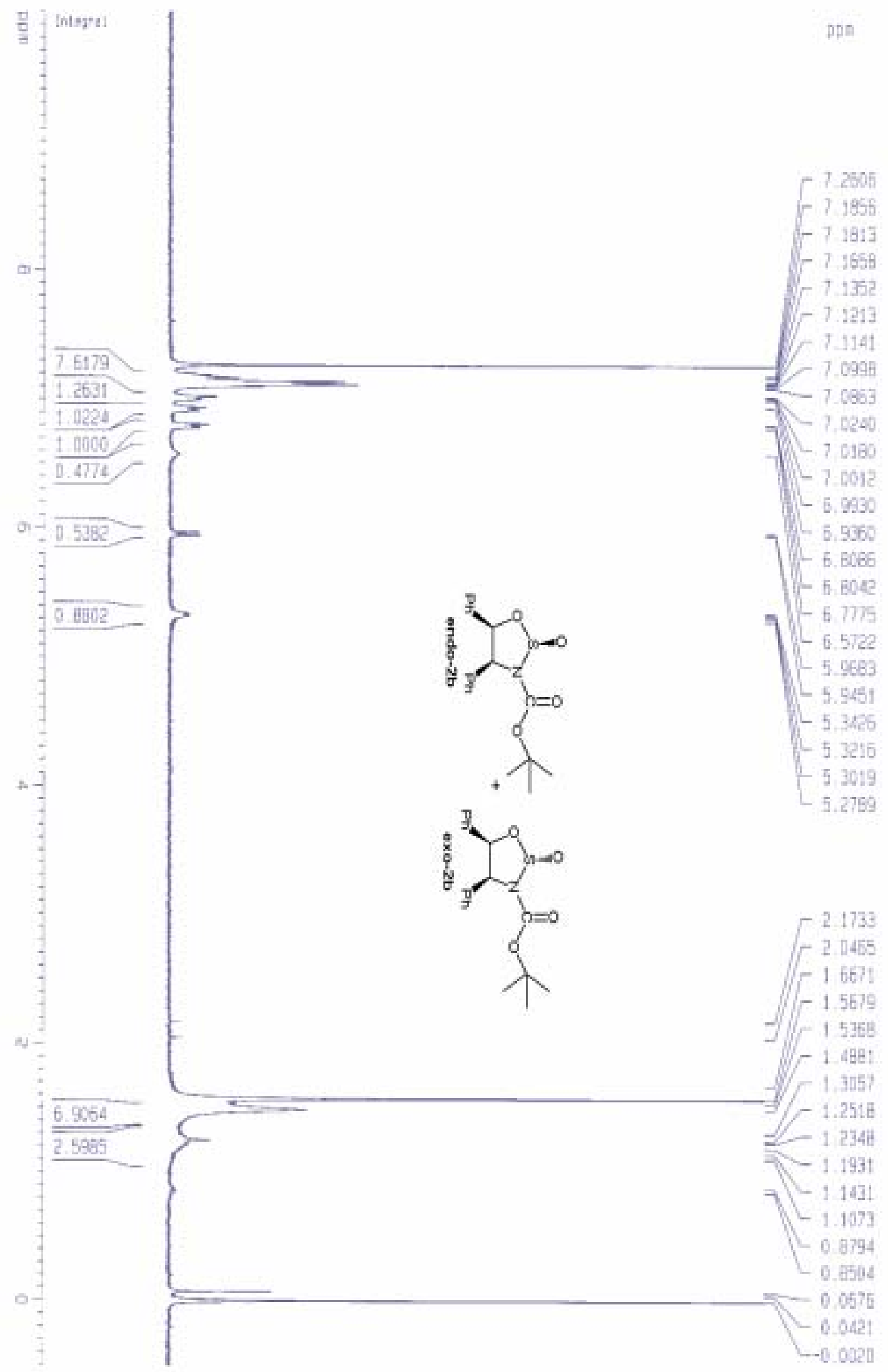




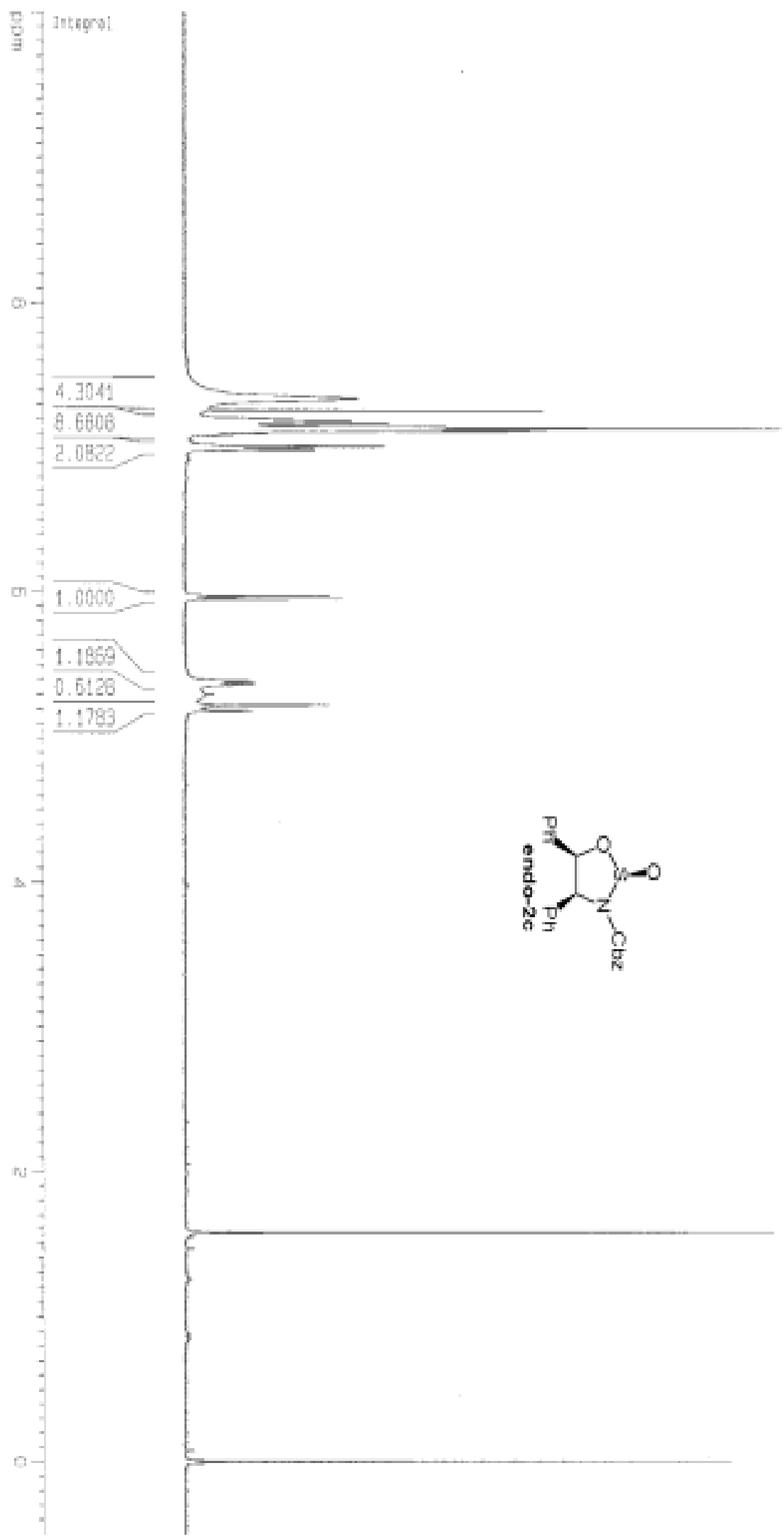

$-7.3410$
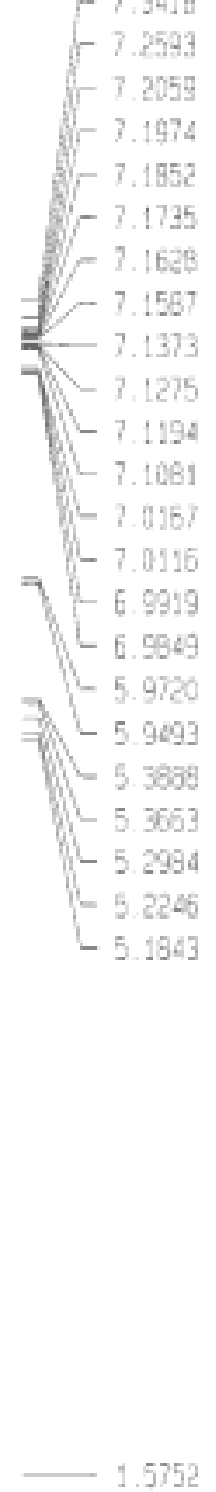

0.0113

$-0.0004$

$-0.0193$ 


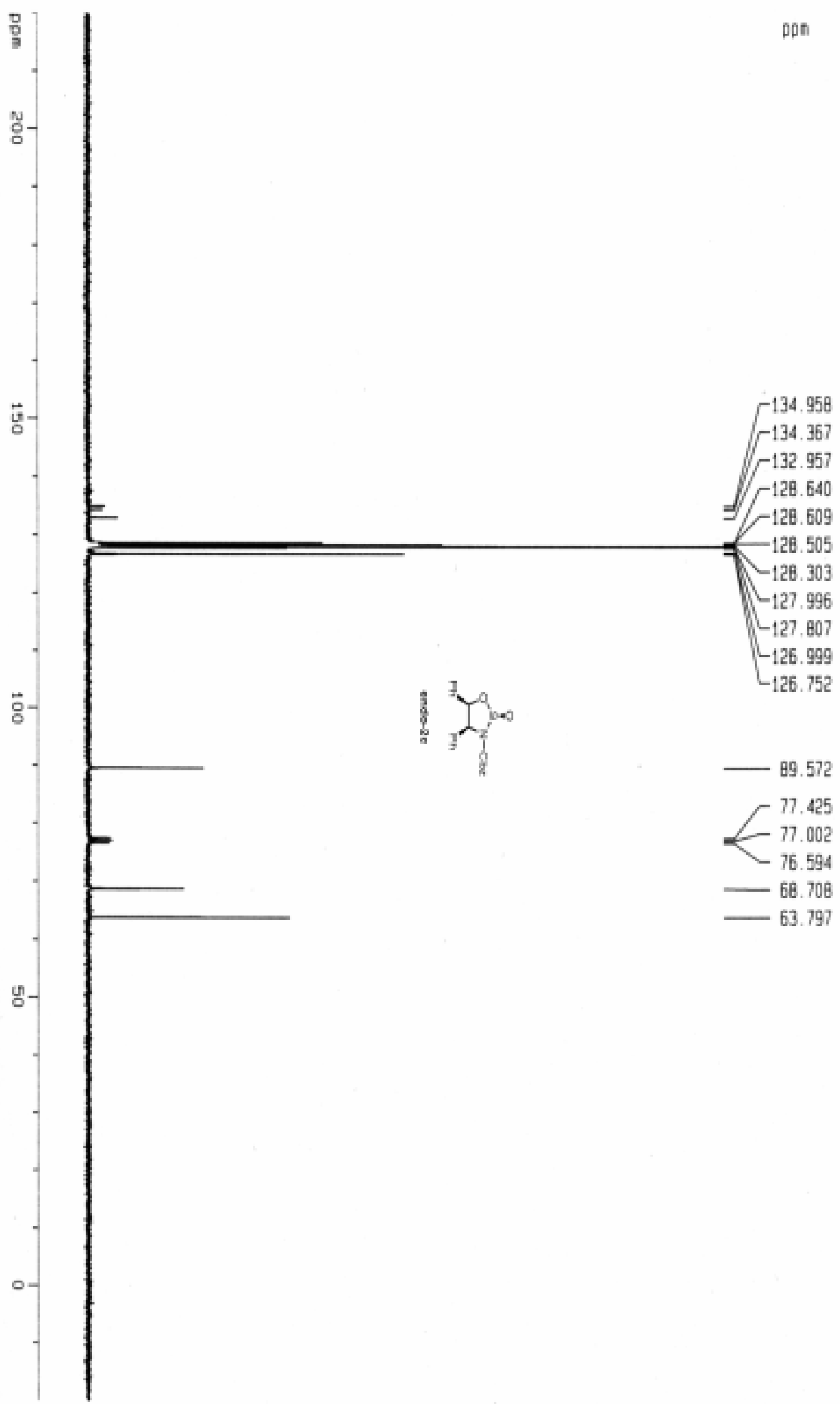



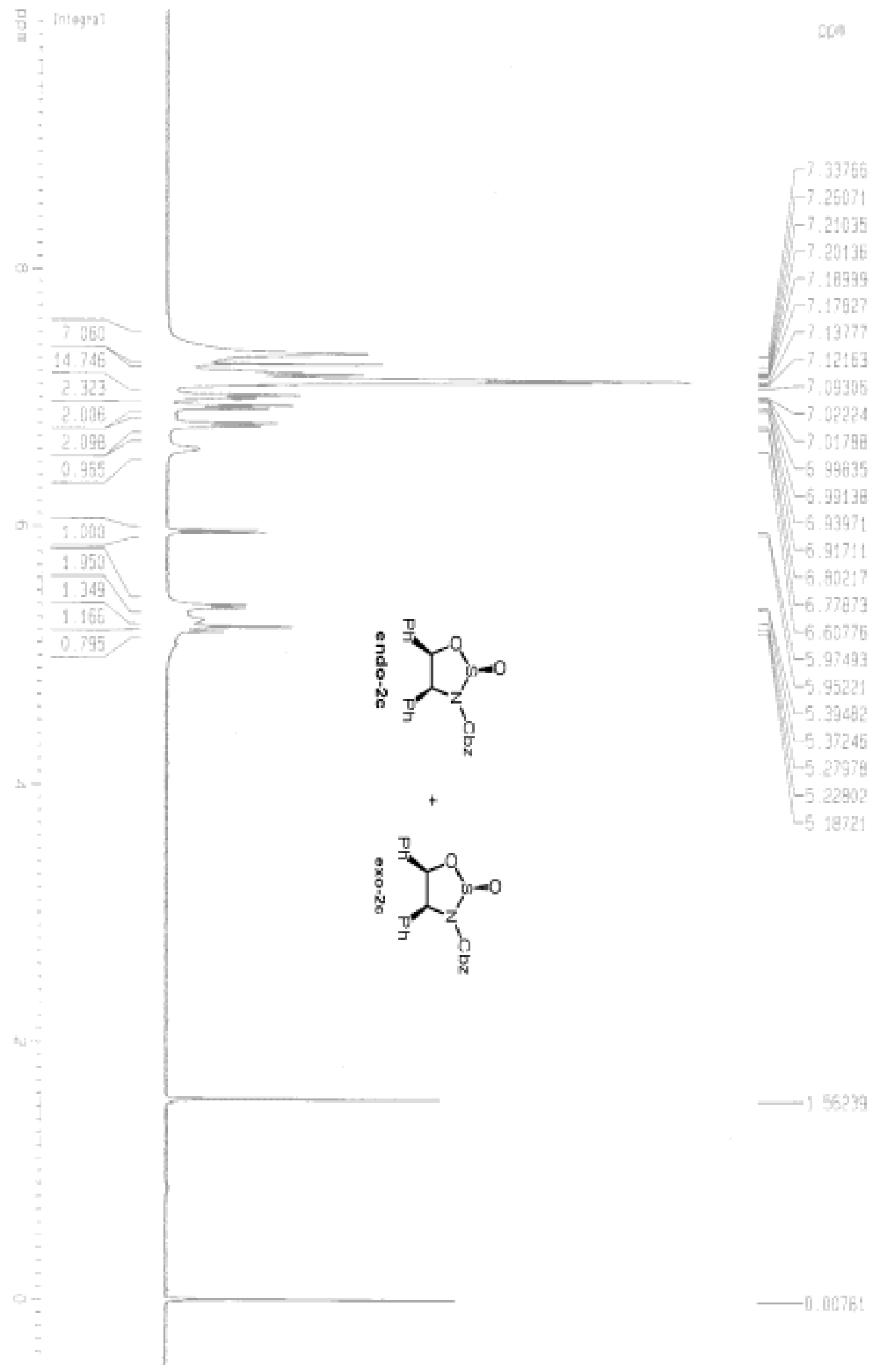


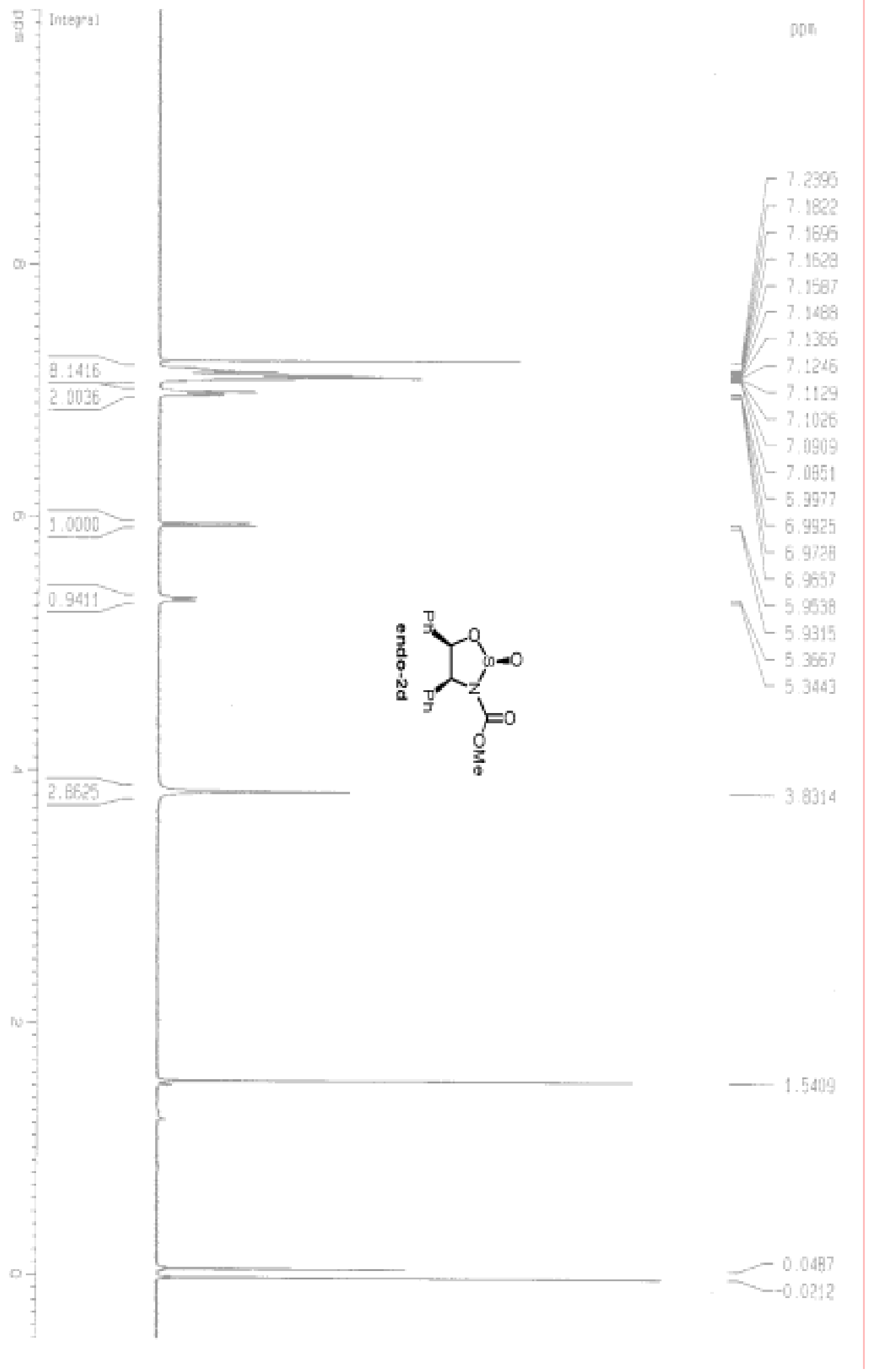




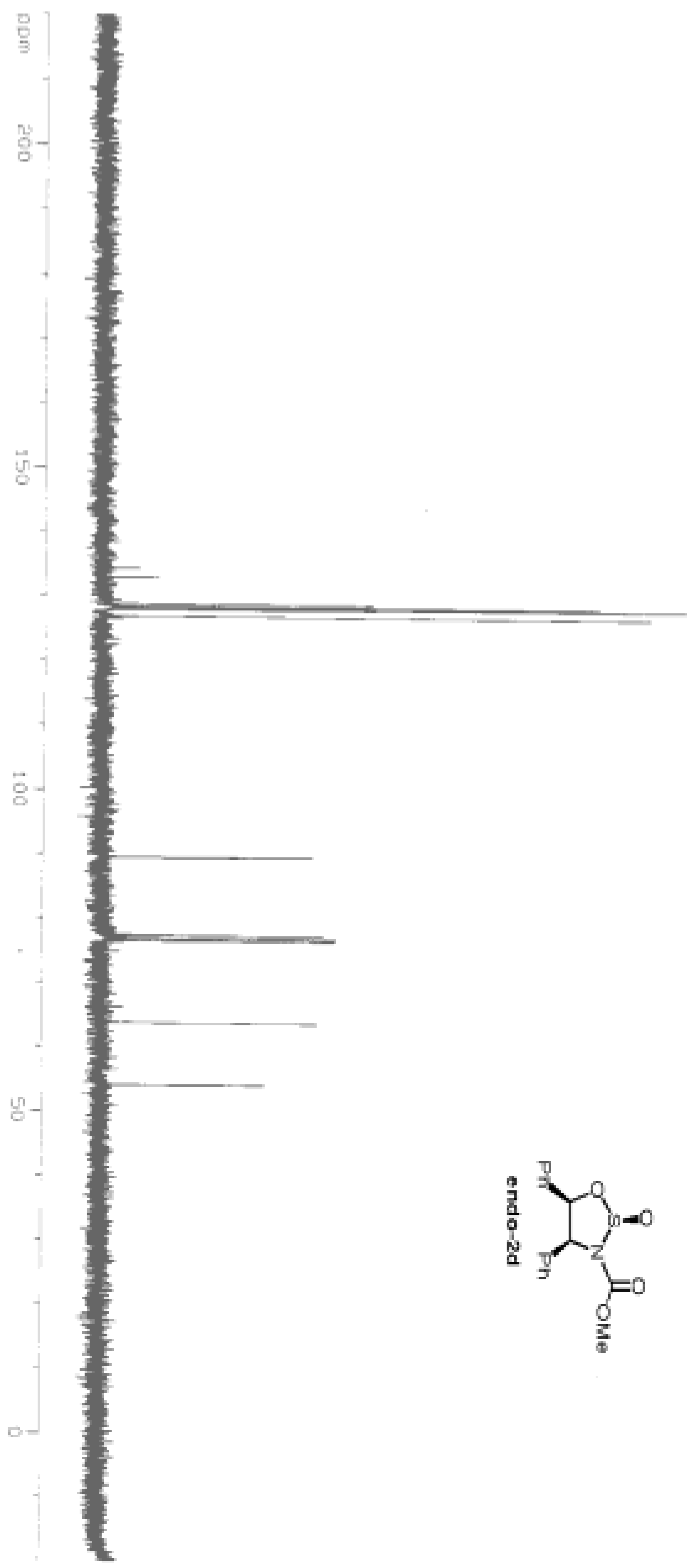

$-134.235$

$-132.693$

$-128.670$

$2-128.290$

$-128.016$

$-123,985$

ᄂ127.93日

$-125.736$

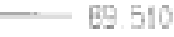

$-77.423$

77.000

- $76.57 \mathrm{E}$

$-53.791$ 


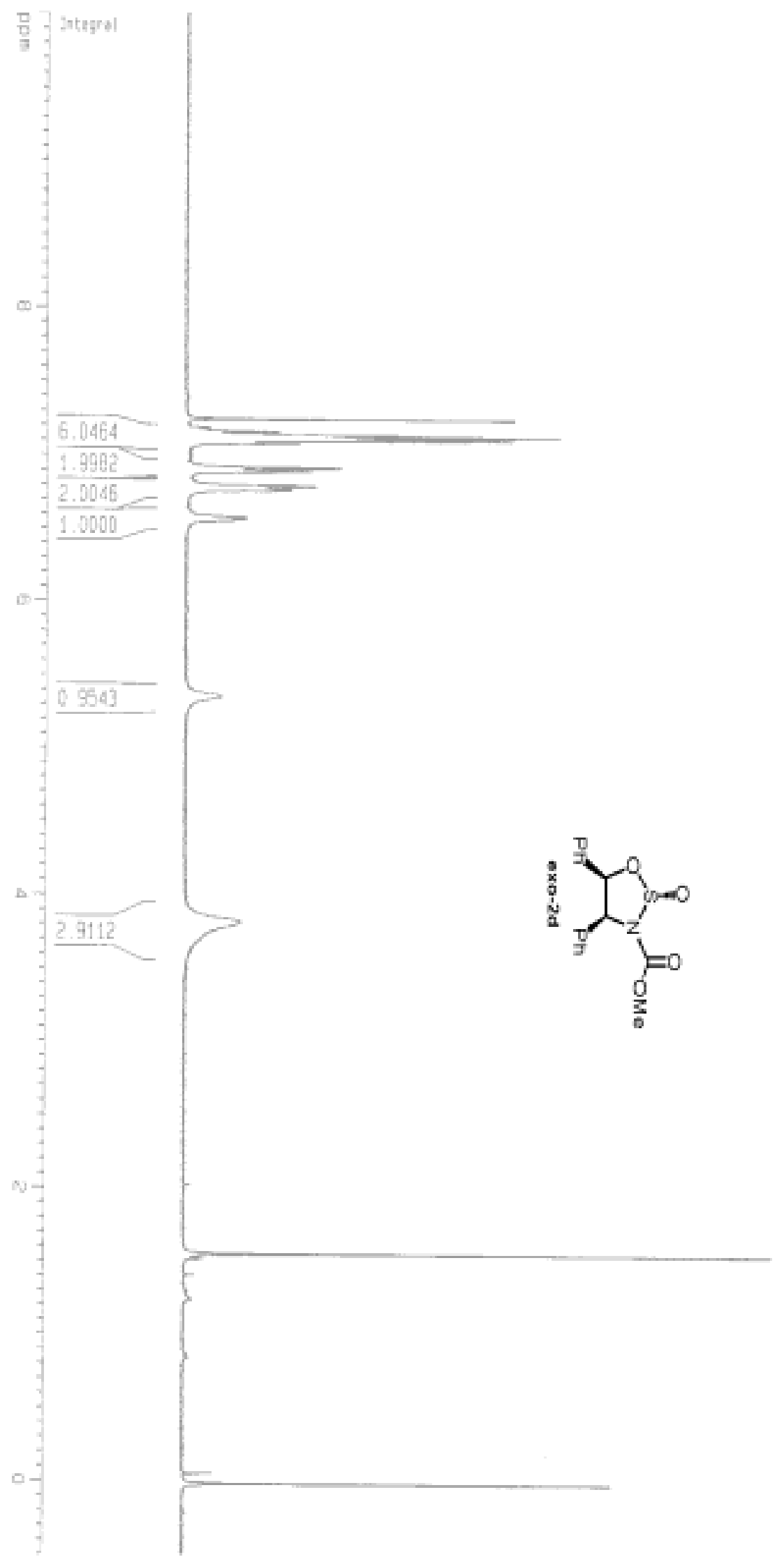

L 5.7873

L 6.766 ?

$-\quad-6.5753$

L 6.5597

$-5.3454$ 


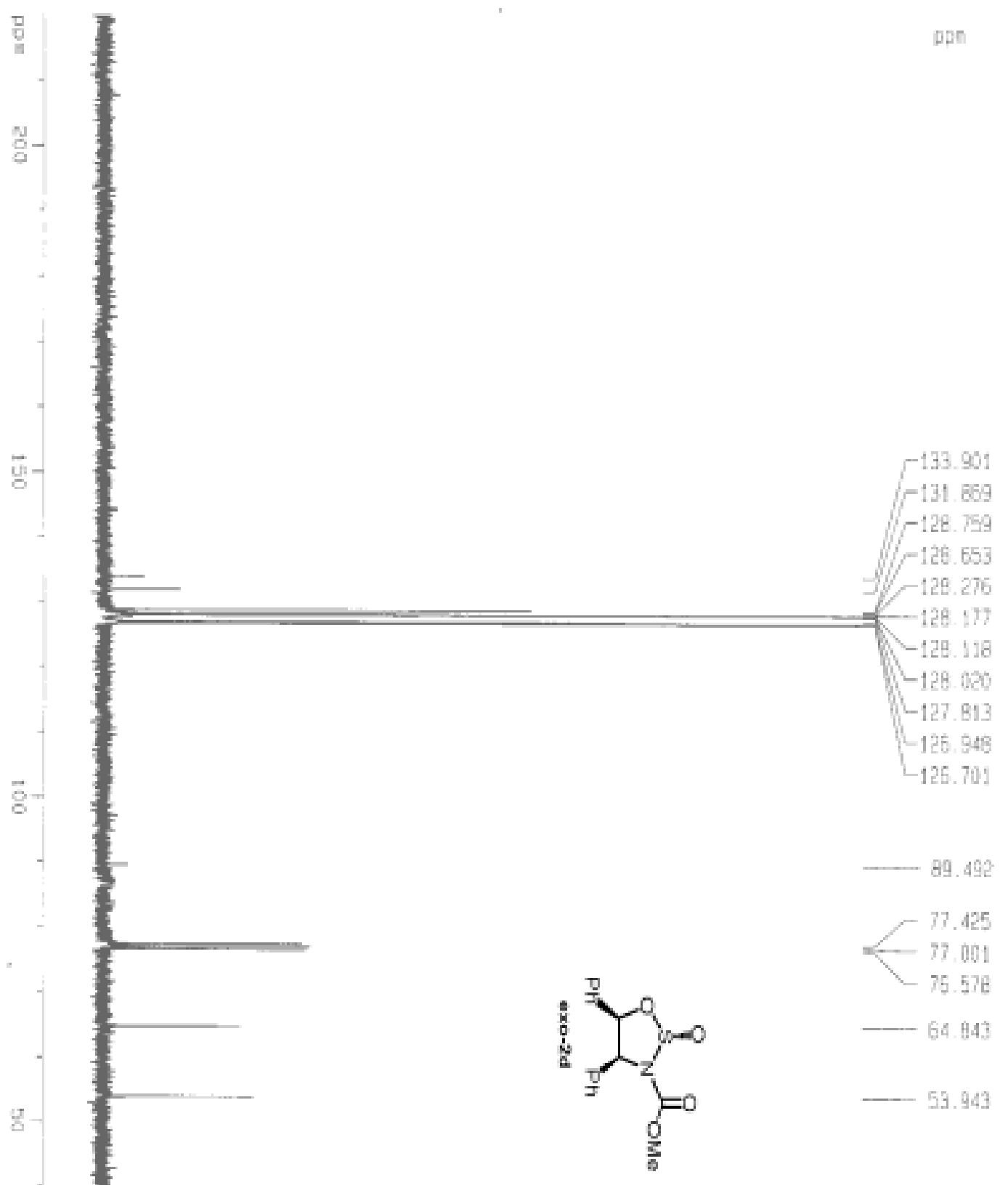




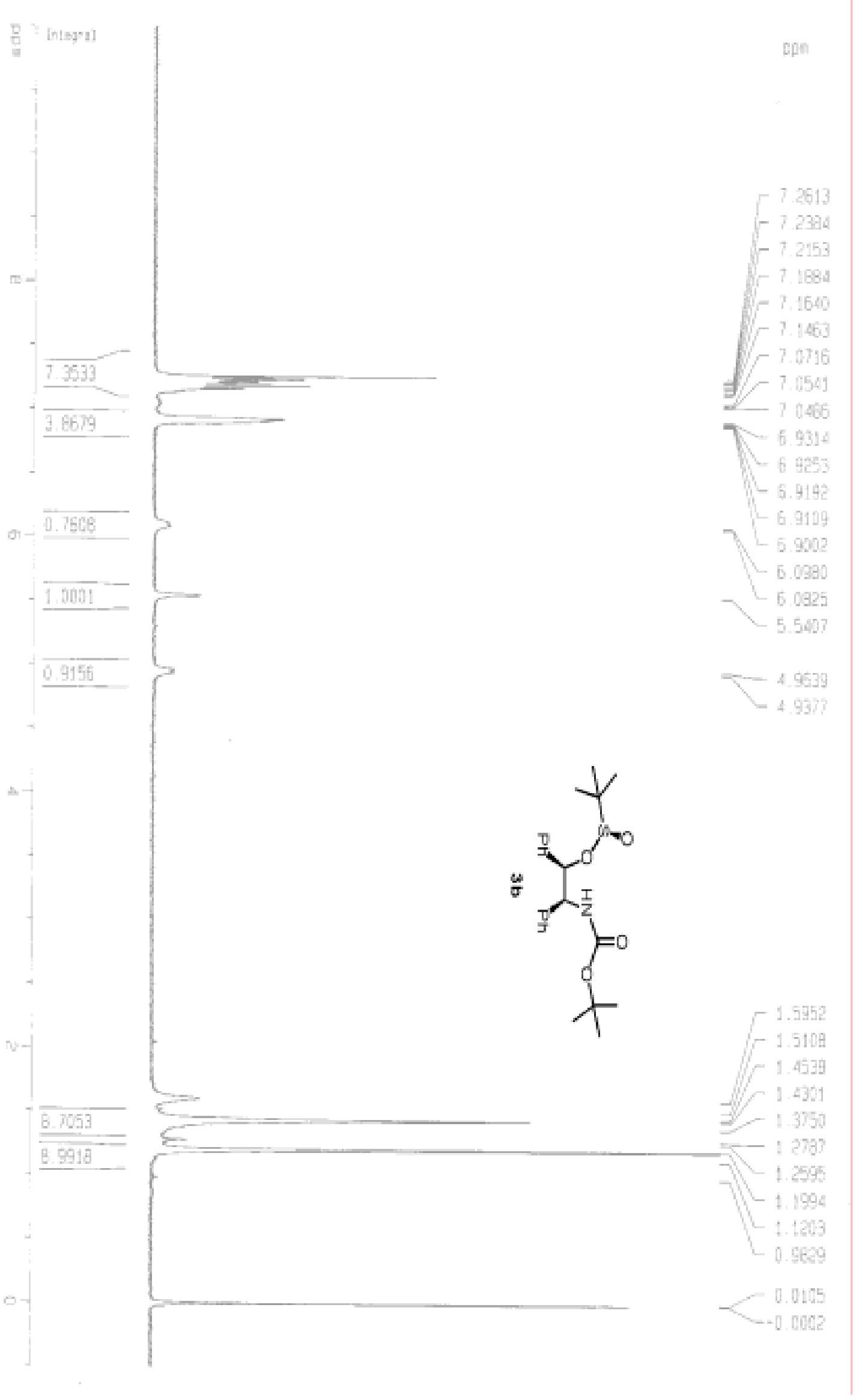



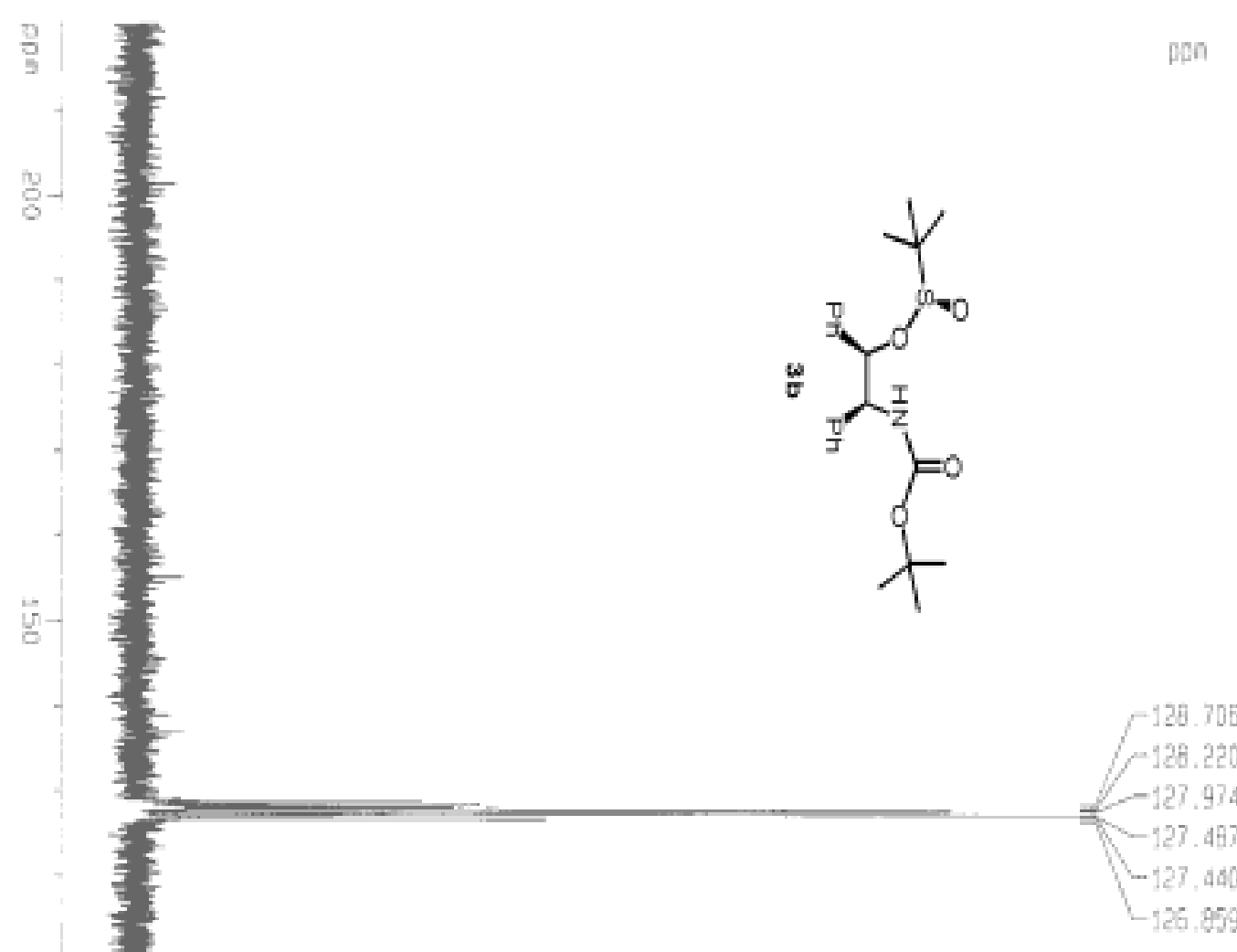

8

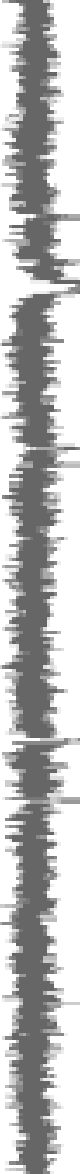

$-84.620$

$-79.674$

77.45

$-77.002$

$-76.578$

58.758
-5071

58.071

g. 


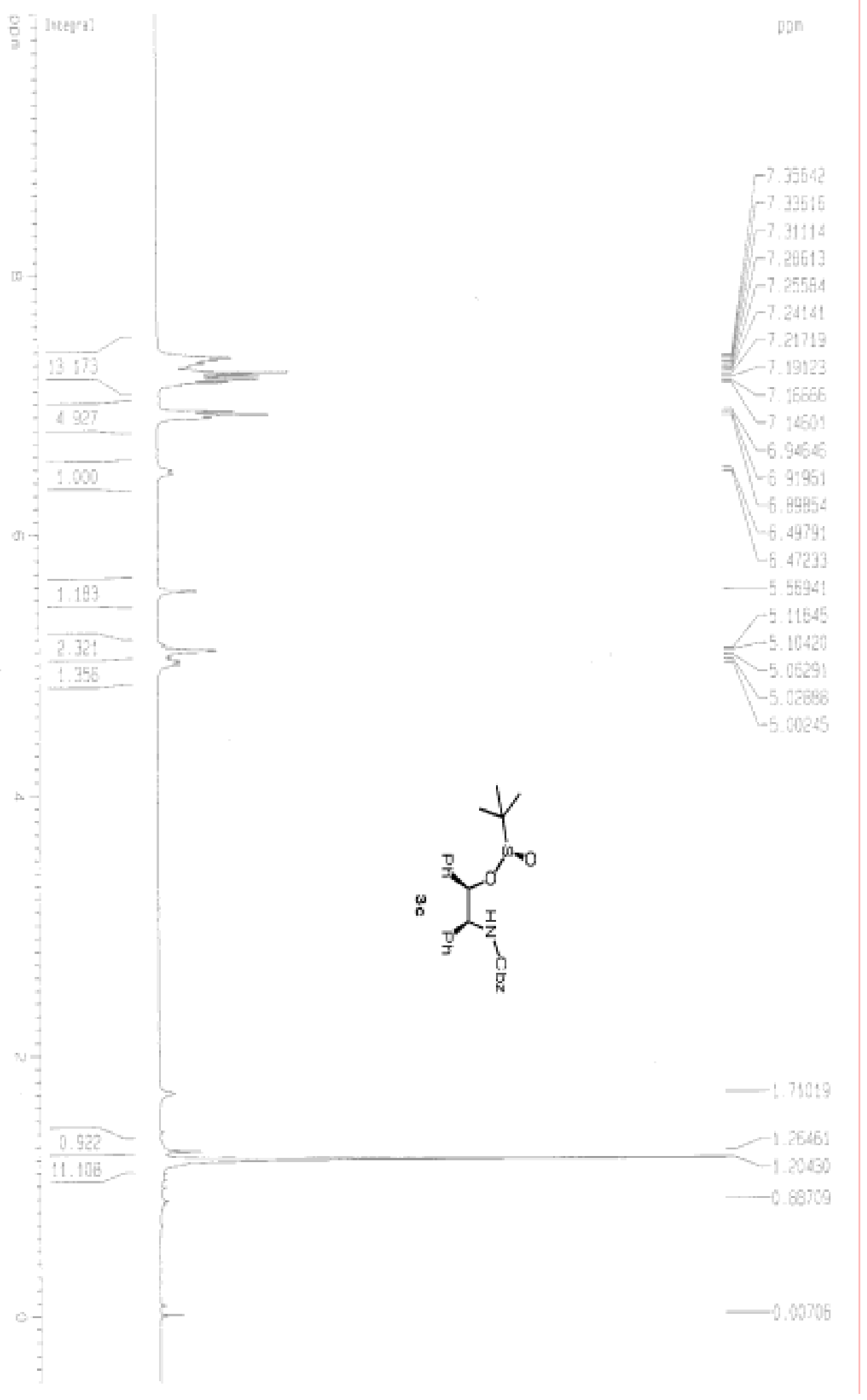




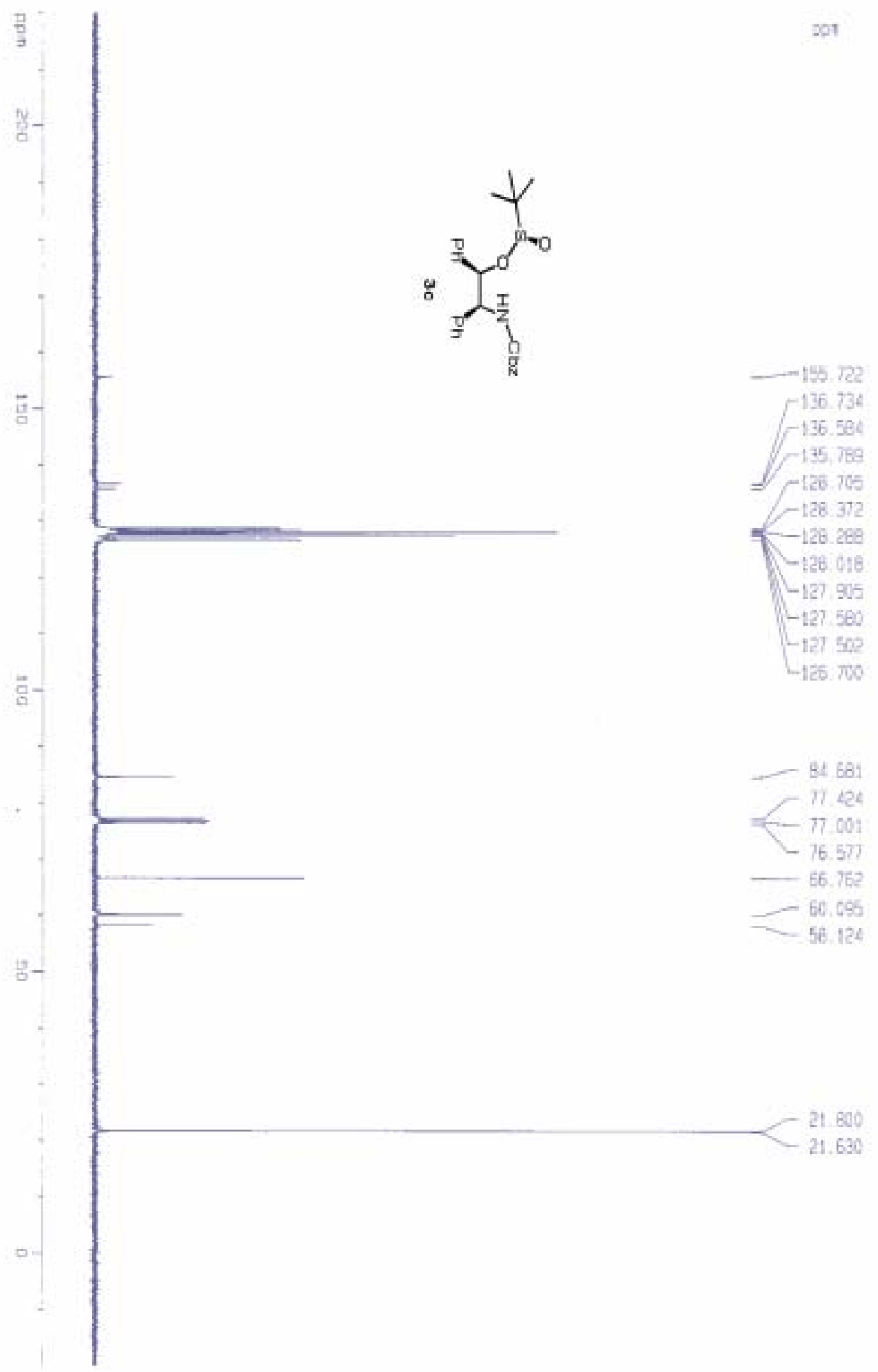




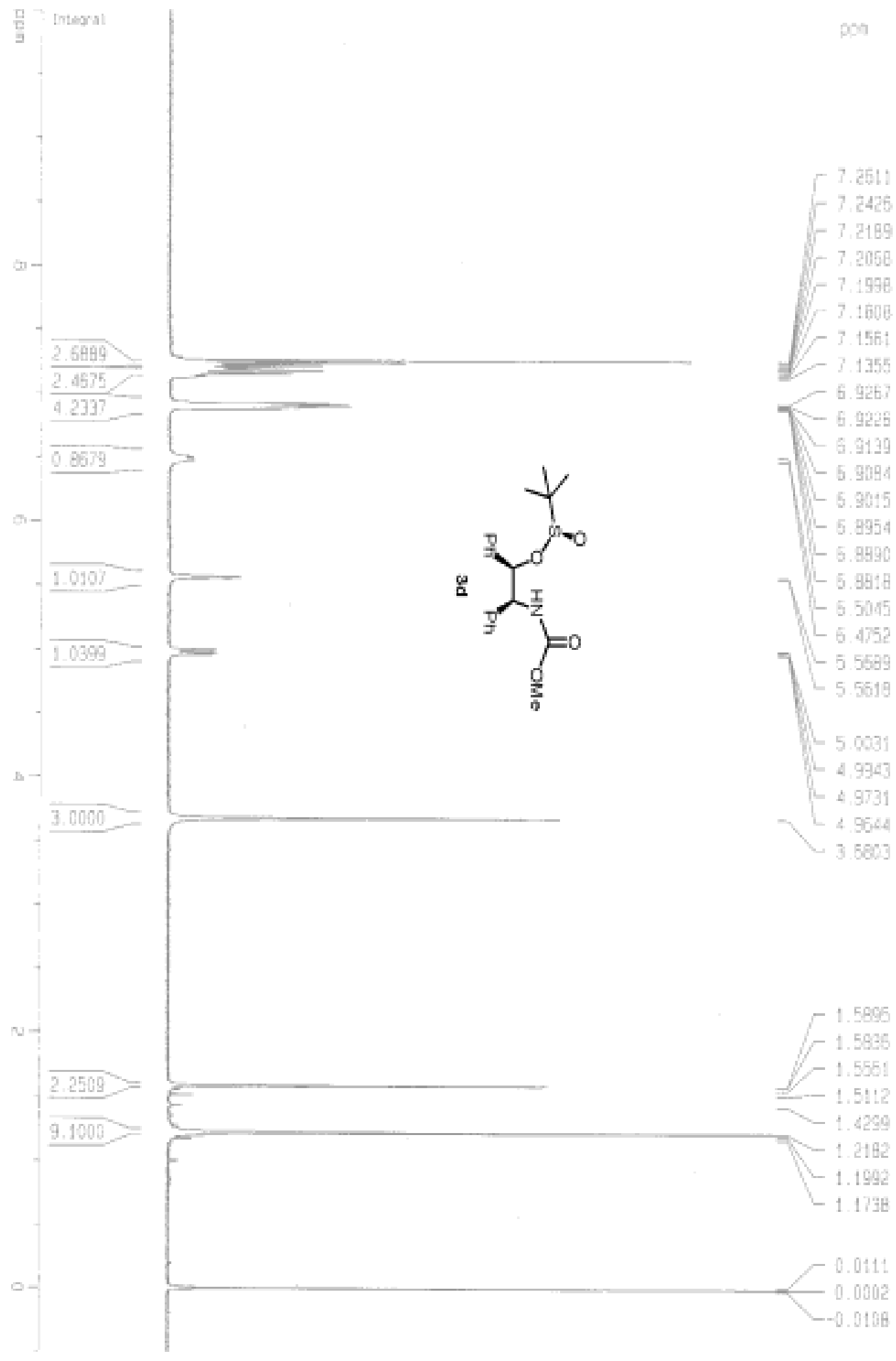




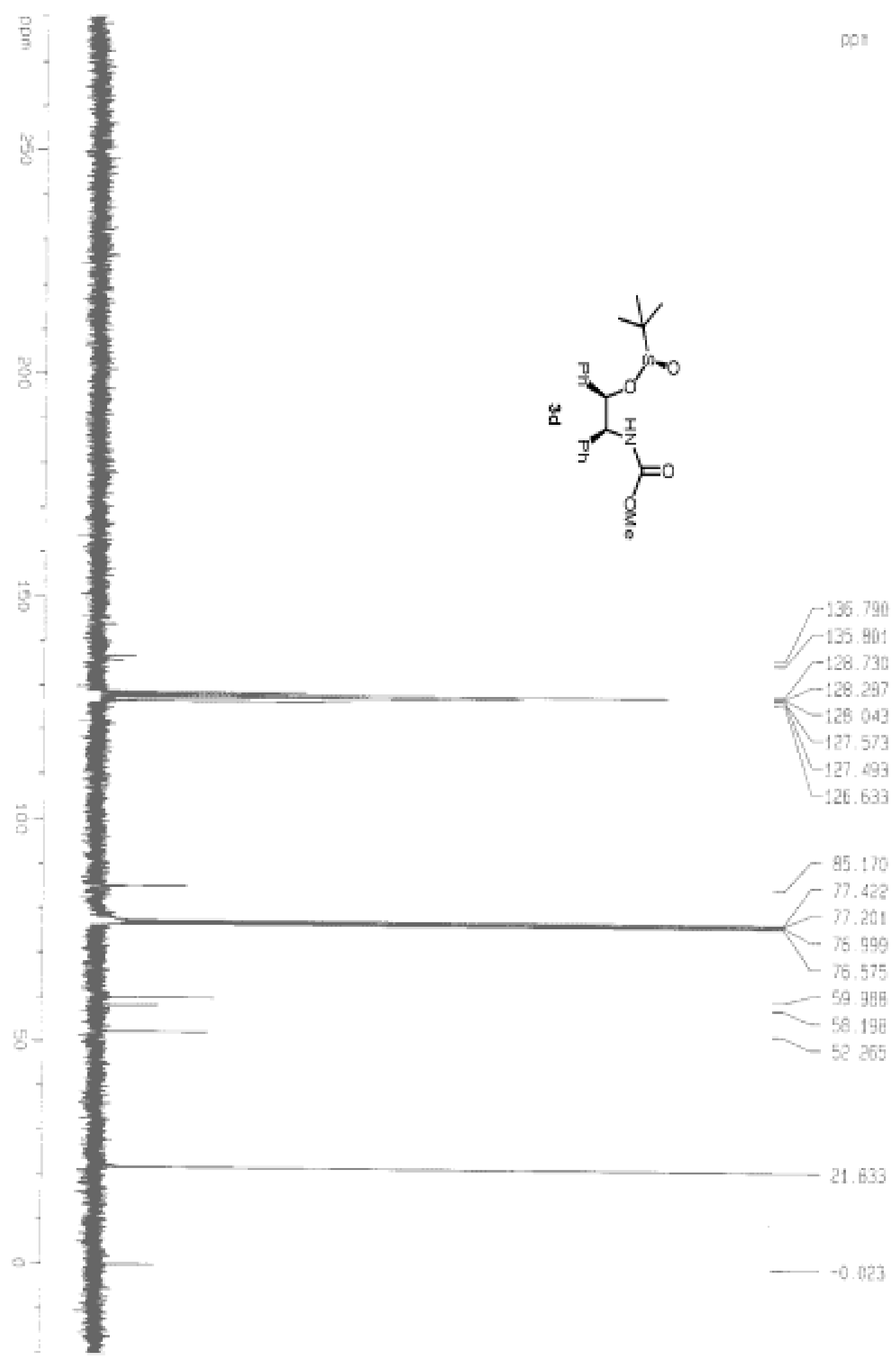



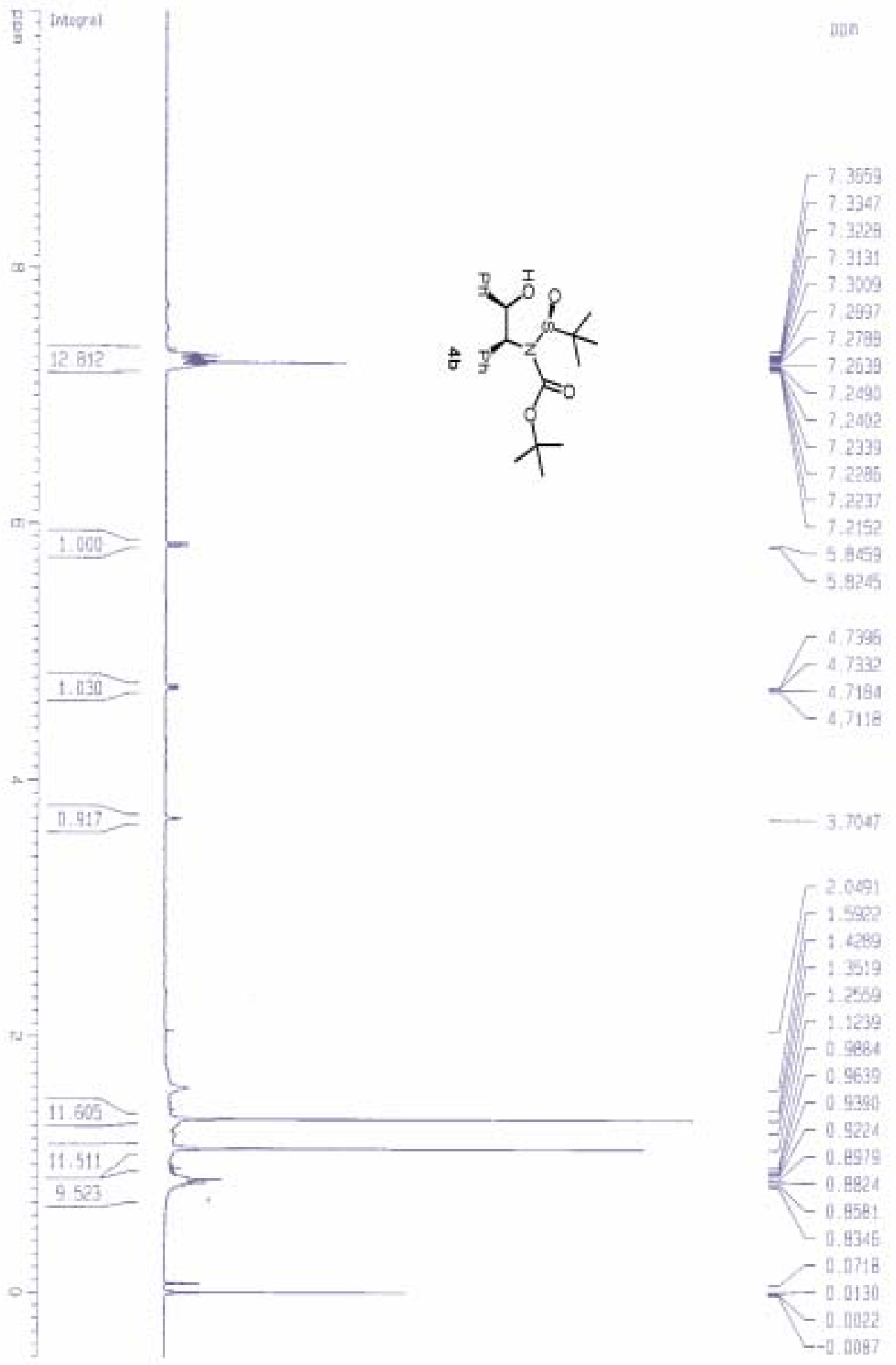


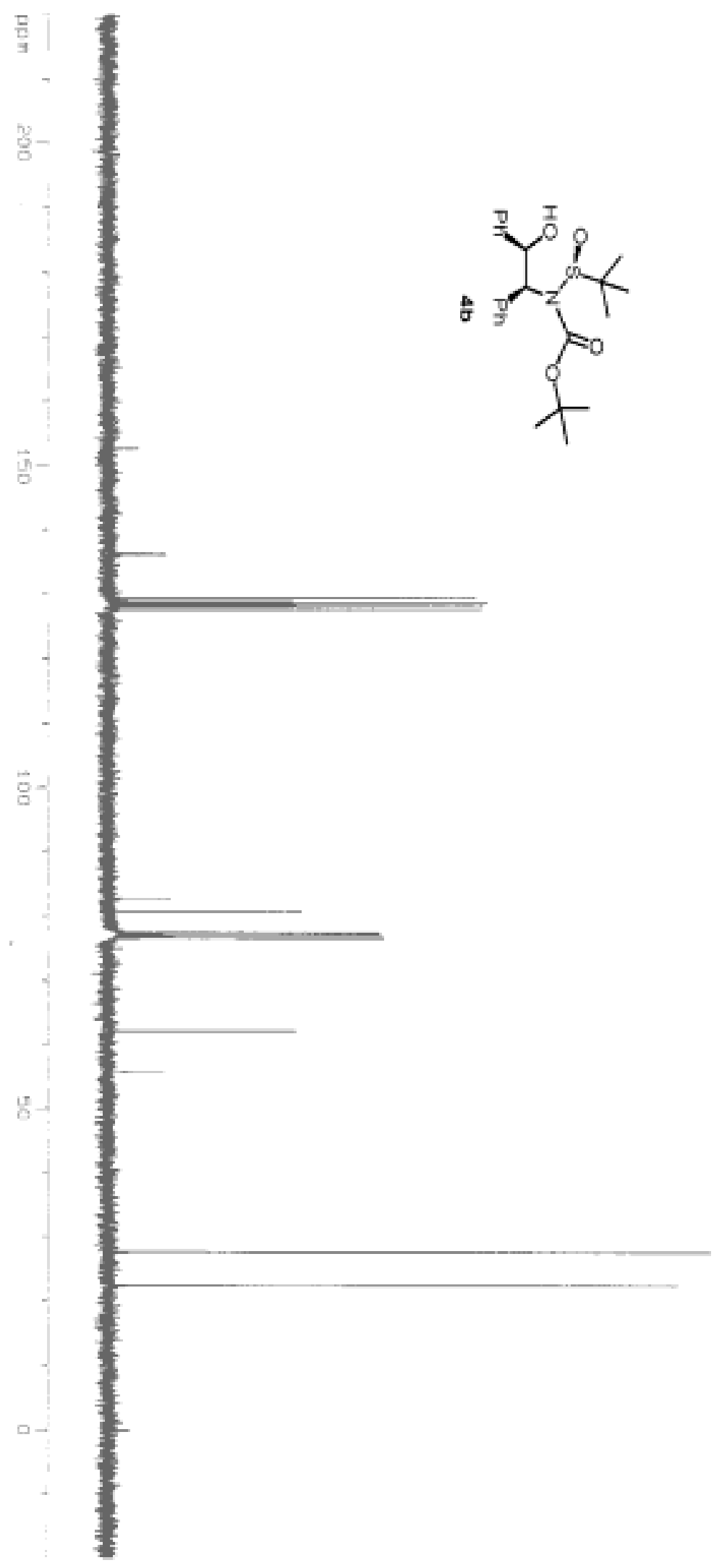




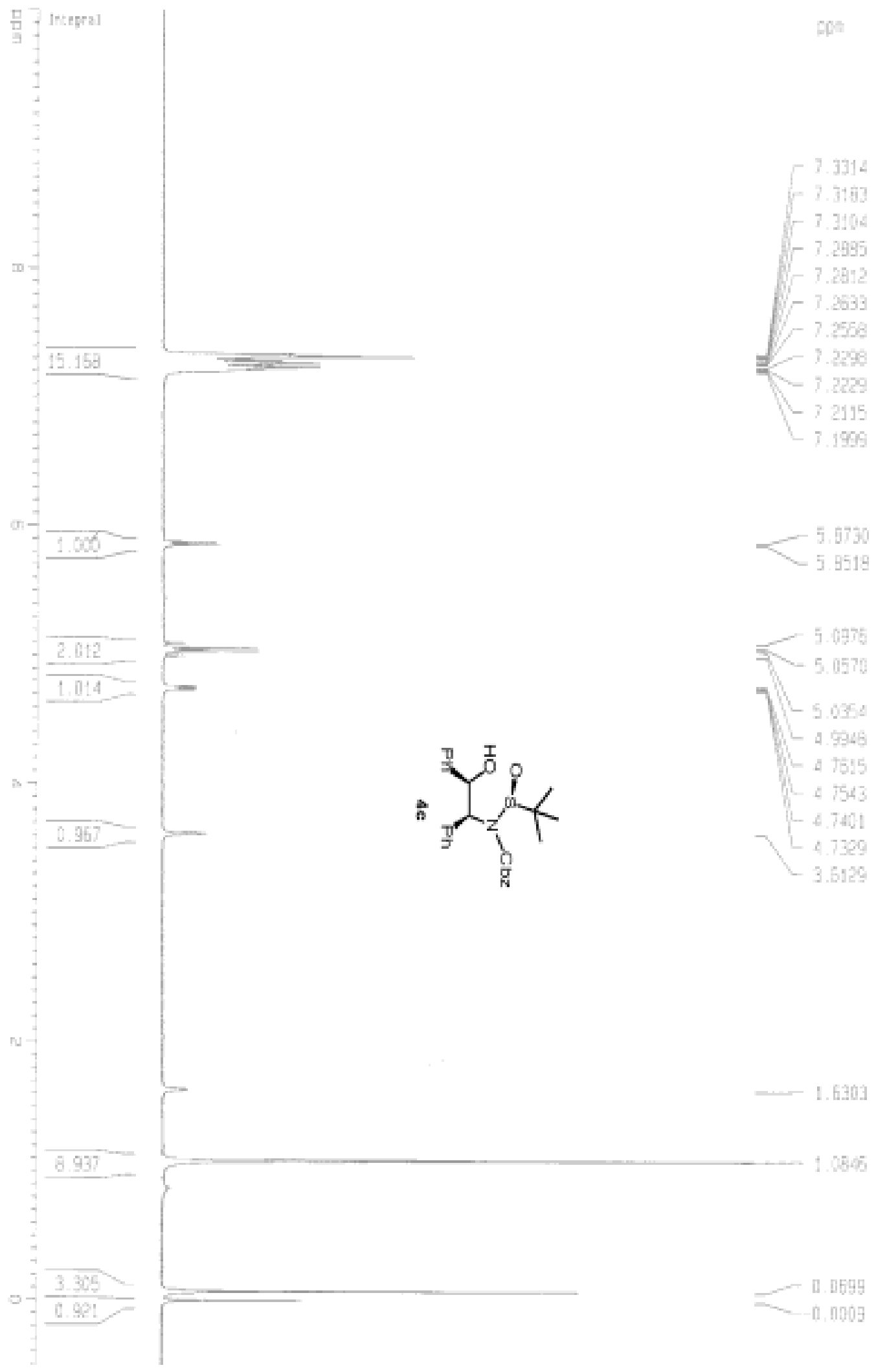




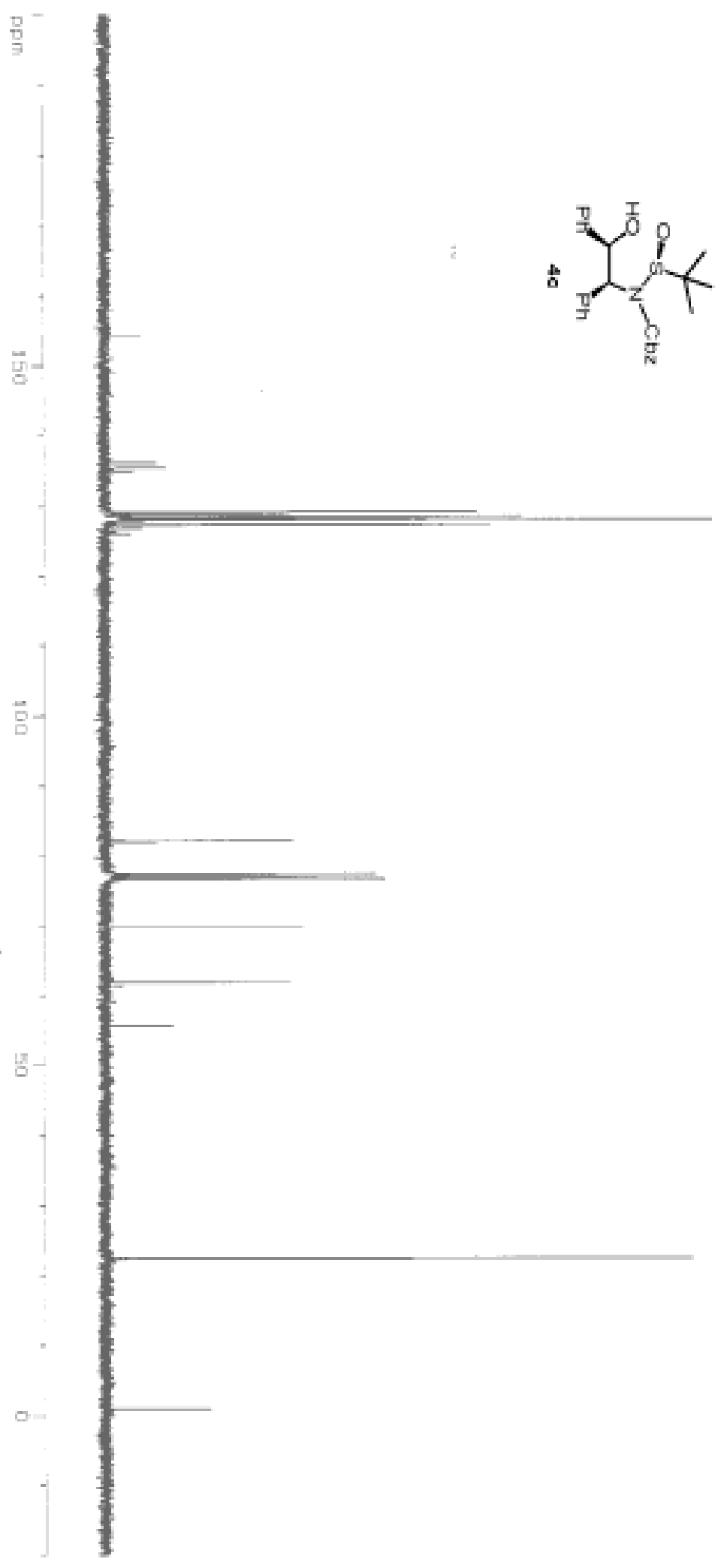

cpn
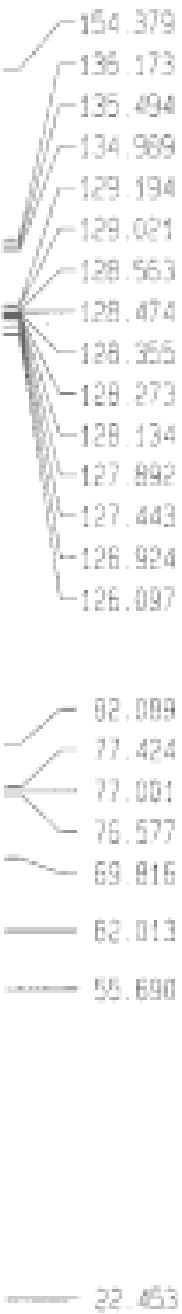


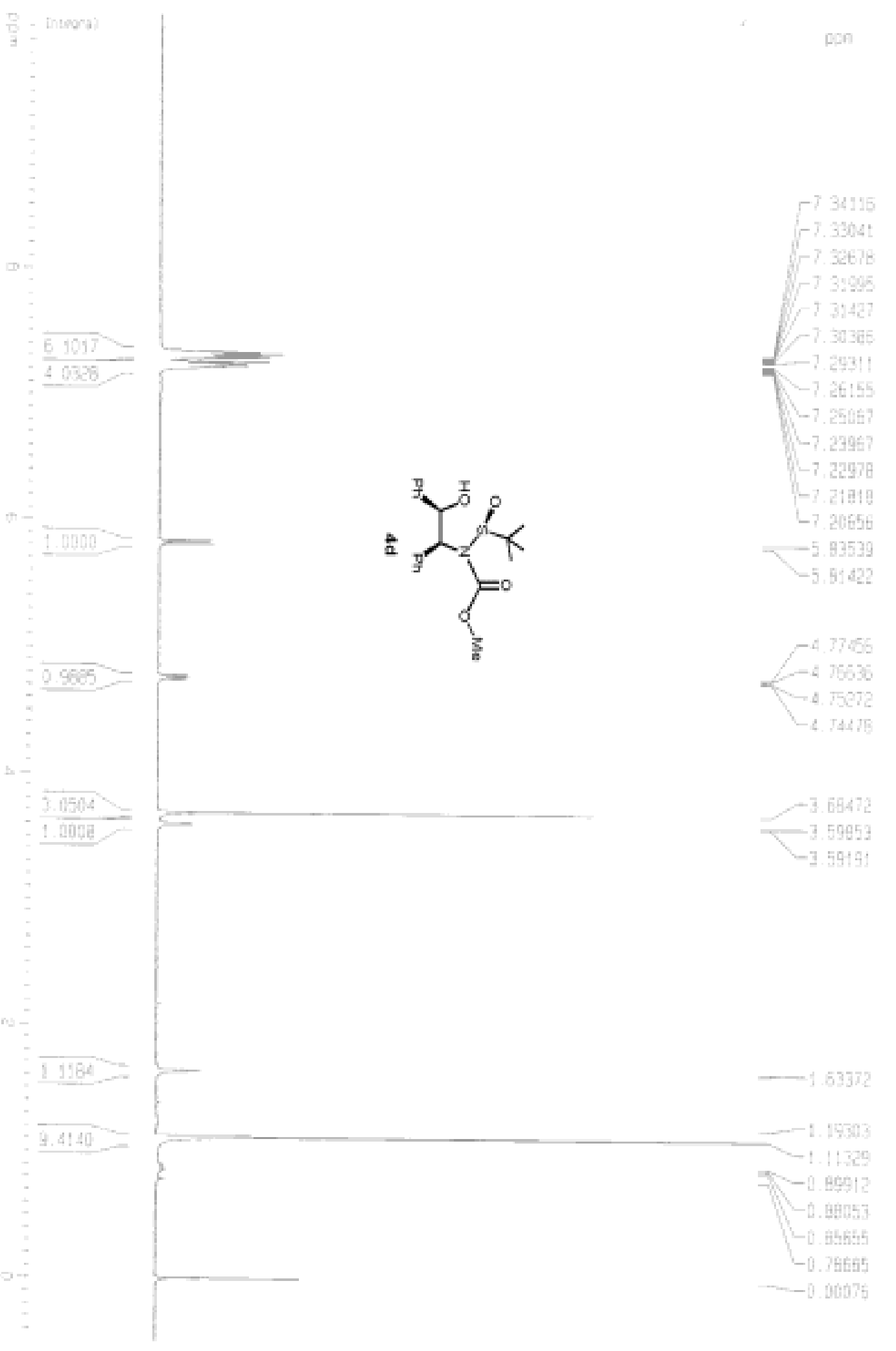




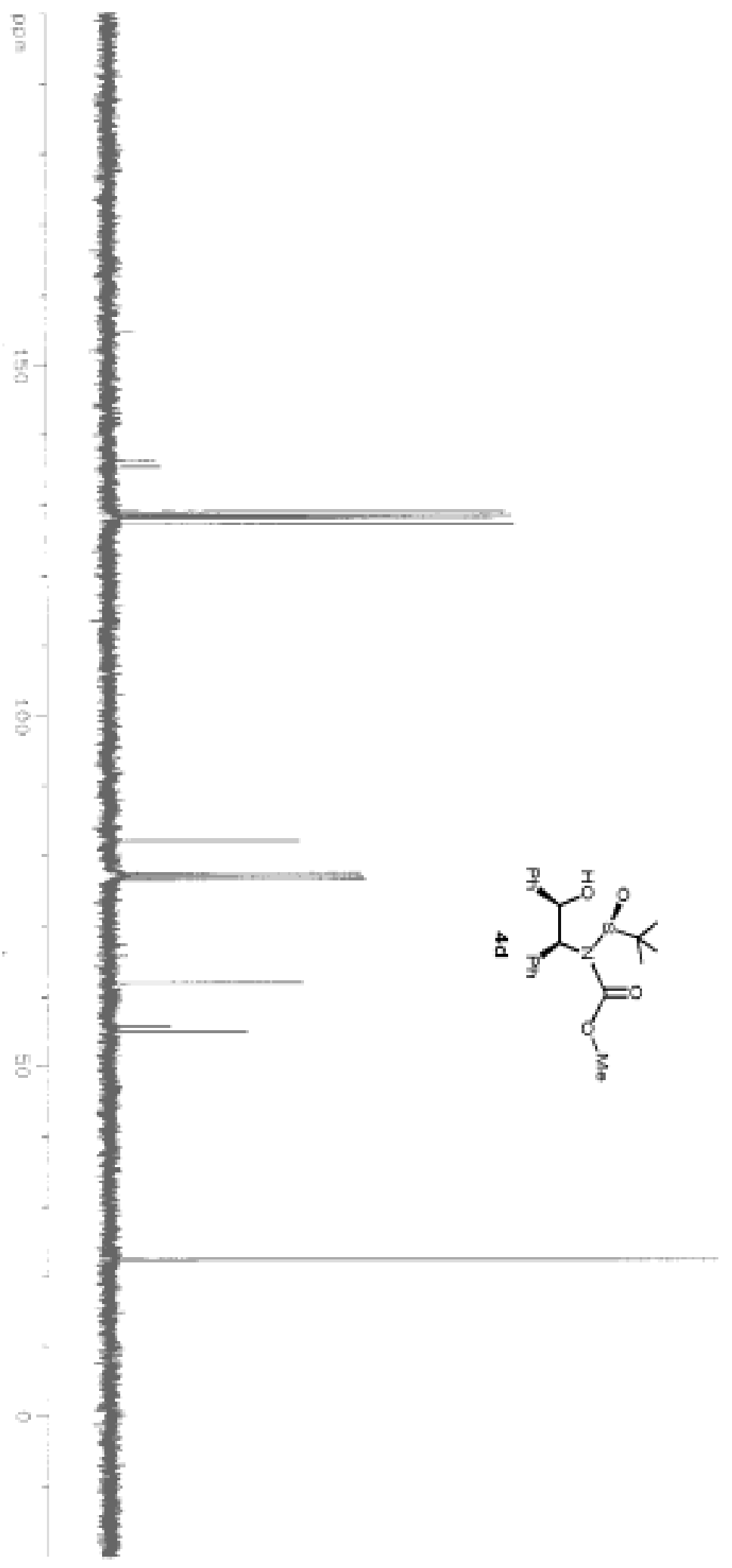

$-135.306$

$-135.477$

$-129.106$

7. 129.037

$-128.579$

$-128.357$

-128.57
-128.183

$-! 27-2\}$

-82.077
-77.42

$-77.423$

75.999

$-76.575$

51.951

55.737

$54.96 \mathrm{~B}$

22.466 


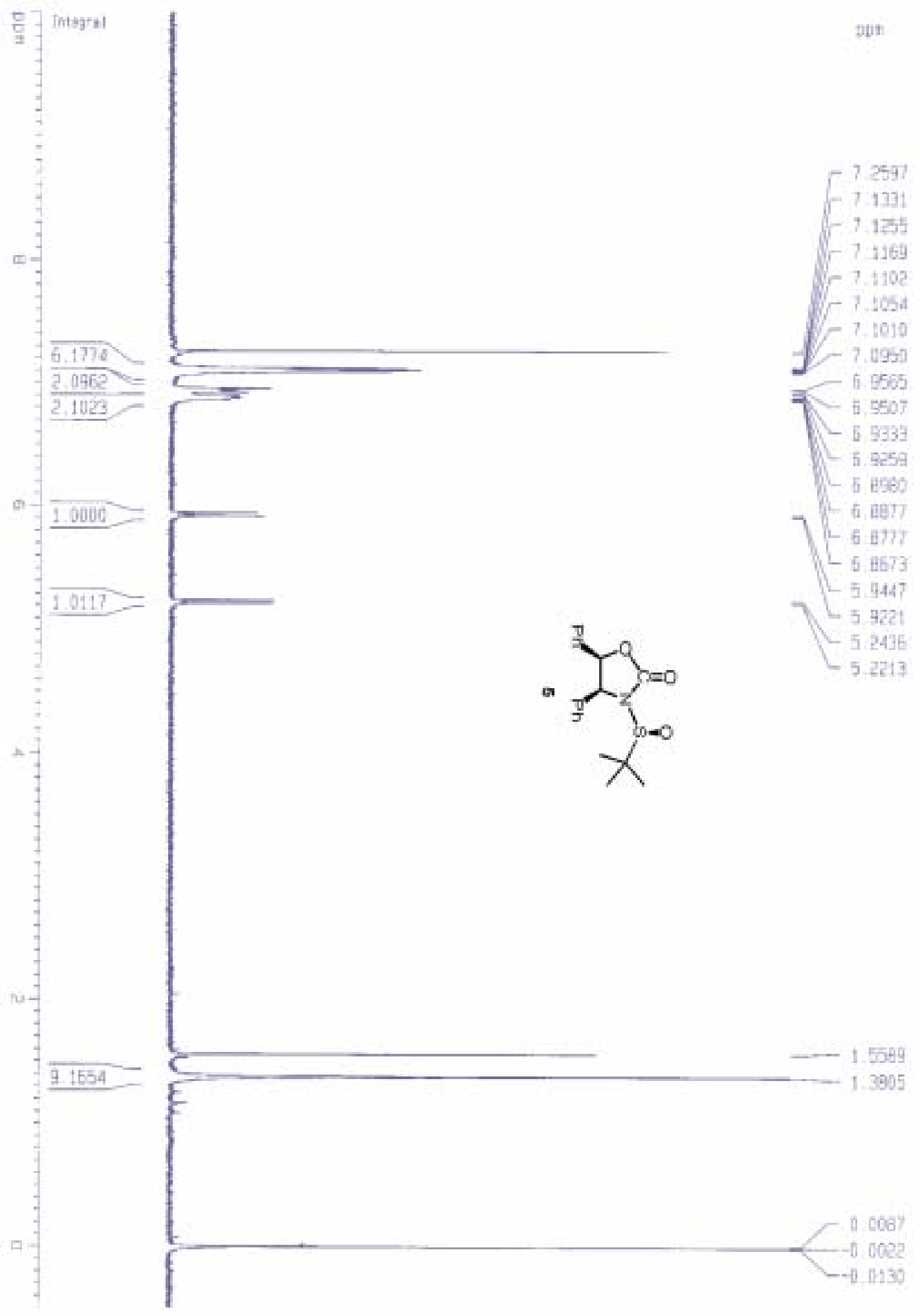




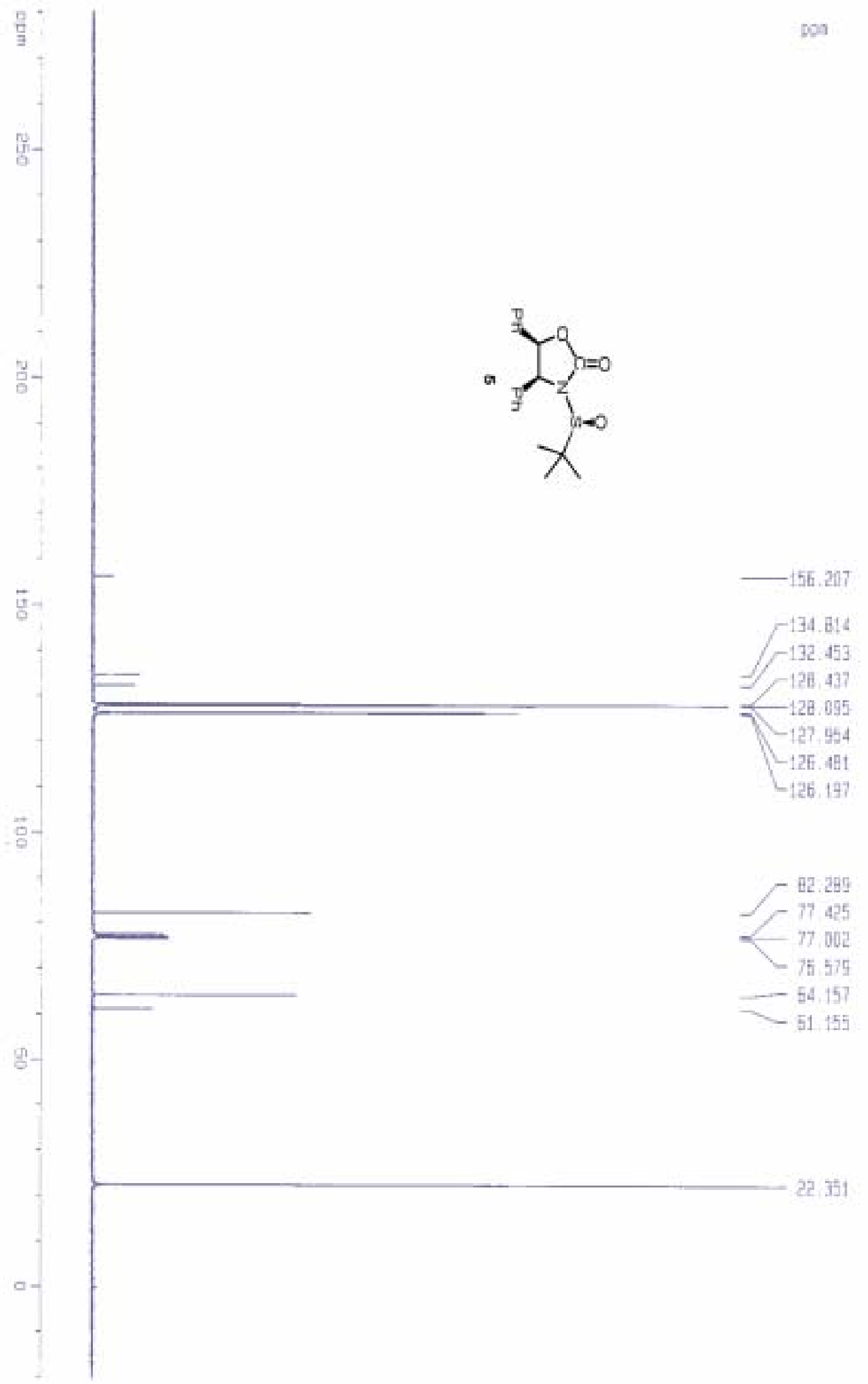




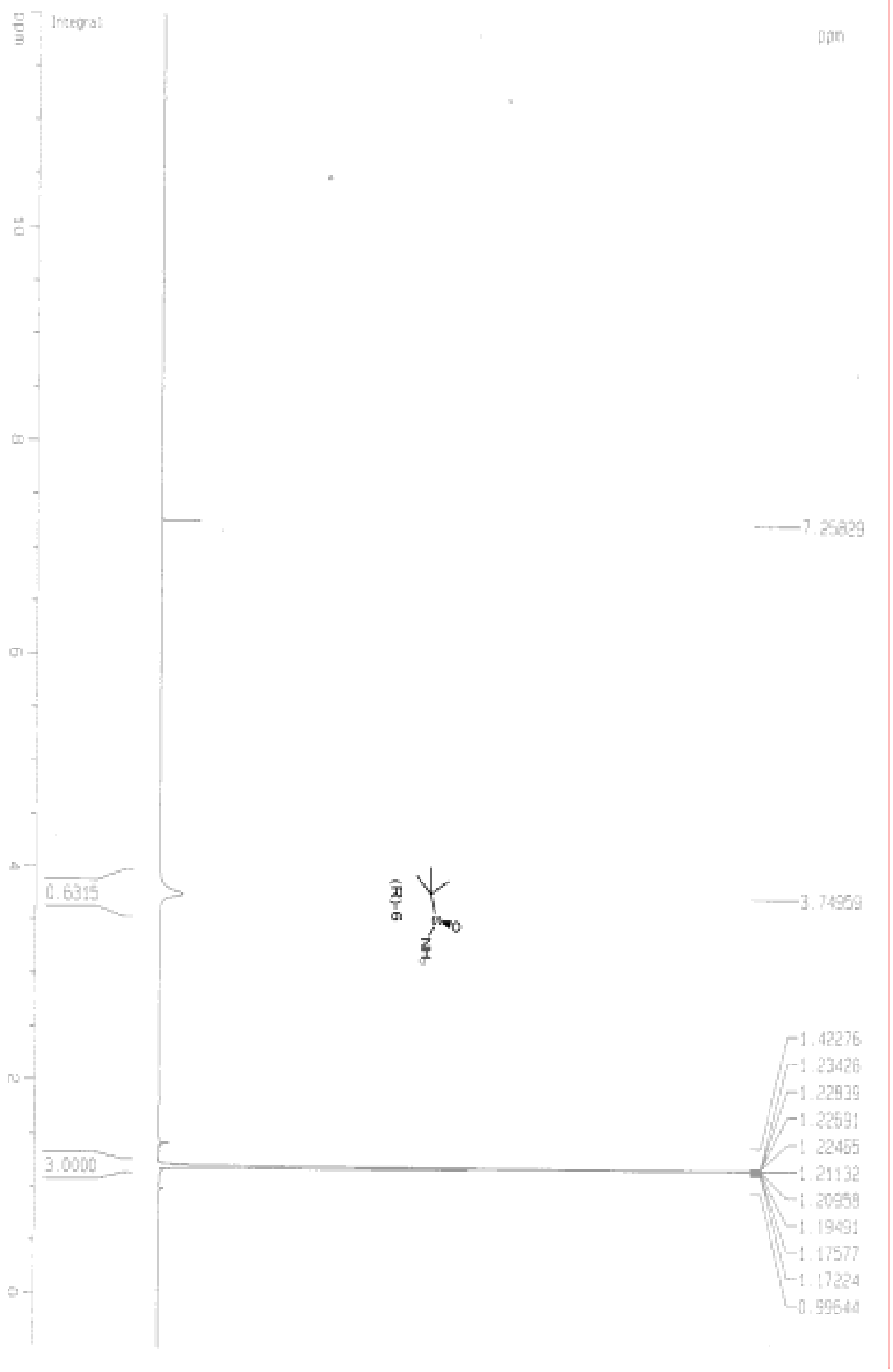


ulas

Project Name: Defauts

Puporled by Leer: System

\begin{tabular}{|c|c|c|c|}
\hline$=$ & $==$ SAMPLE & NFORMA T & $10 \mathrm{~N}$ \\
\hline Semple Name: & wch-1 & Acoured Br & System \\
\hline Sample Type: & Unknown & Date Acquired: & $6 / 29 / 0411: 0553 \mathrm{AM}$ \\
\hline Va: & 1 & Acq. Mthod: & 1004222 \\
\hline triection a. & 2 & Dats Processed: & 6/2904 11:24:56 AM \\
\hline riection Volume. & 20.00 ul & Channel Name: & 2487Charnel 1 \\
\hline Ren Time: & 180.00 Mrrules & Sample Sel Nare: & \\
\hline
\end{tabular}

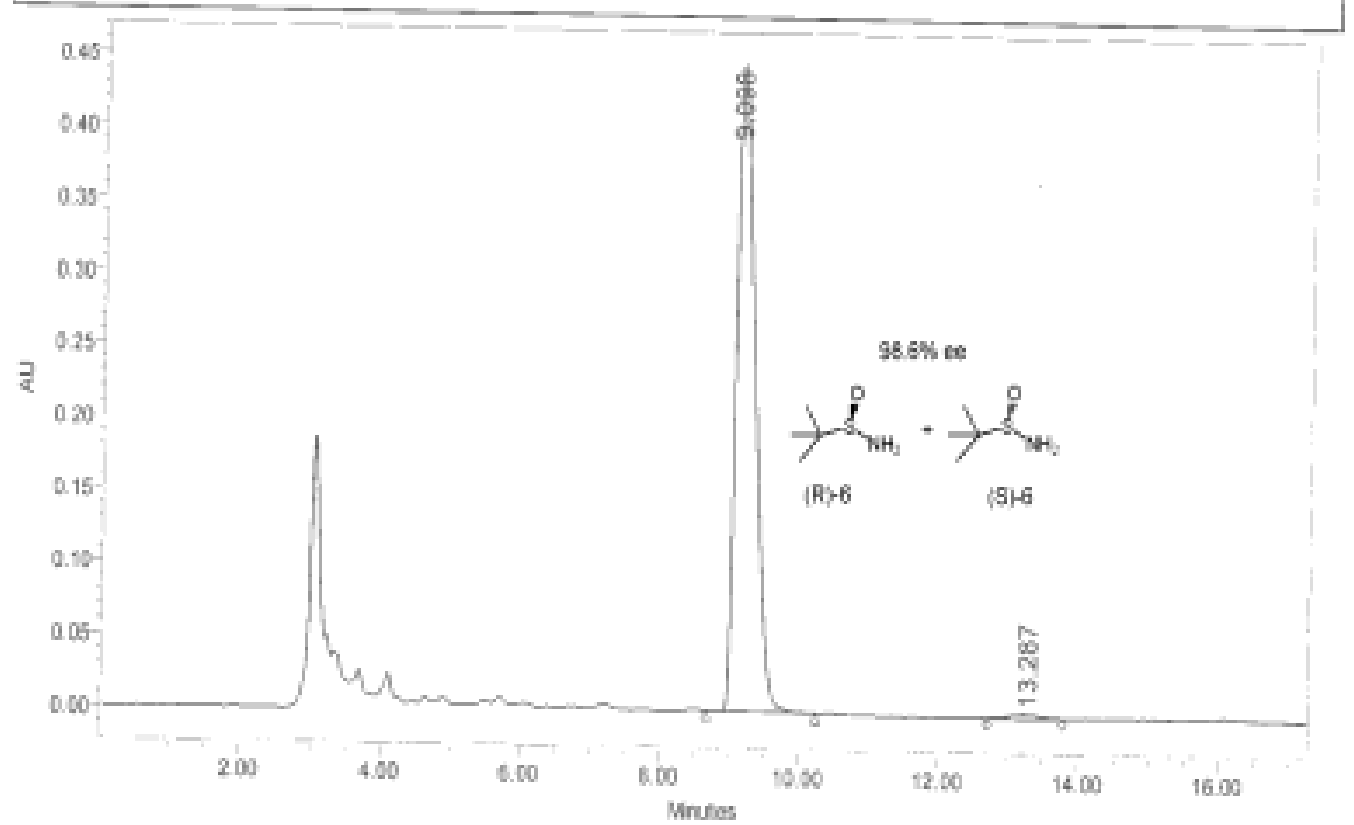

\begin{tabular}{|c|c|c|c|c|c|}
\hline & $\begin{array}{c}R T \\
(\mathrm{nn})\end{array}$ & $\begin{array}{c}\text { Ares } \\
\left(\mu N^{\prime} \text { sec }\right\rangle\end{array}$ & \% Area & $\begin{array}{c}\text { Feigt } \\
\langle(N)\end{array}$ & $\begin{array}{c}\% \\
\text { Height }\end{array}$ \\
\hline 1 & 9.096 & 8137503 & 98.66 & 444754 & 99.28 \\
\hline 2 & 13.287 & 110689 & 1.34 & 3241 & 0.72 \\
\hline
\end{tabular}


ulas

Projact Nare: Defauts

Reported by User. Systam

\begin{tabular}{|c|c|c|c|}
\hline \multicolumn{2}{|c|}{ SAMPLE } & \multicolumn{2}{|c|}{ INFORMATION } \\
\hline Sample Name: & wch.2 & Acquired By. & Syatem \\
\hline Somple Type: & Unknown & Dale Acoquired: & $6 / 290411.26 .17 \mathrm{AM}$ \\
\hline Vist & 1 & Acc, Mathod & ing \\
\hline hịecton t. & 3 & Dete Processect & G:2904 11:43:54 AM \\
\hline hectan Volume: & $20.00 \mathrm{u}$ & Channel Name: & 2487Chaned 1 \\
\hline Pun The & 180.00 Mndes & Sarmple Set Nerrec & \\
\hline
\end{tabular}

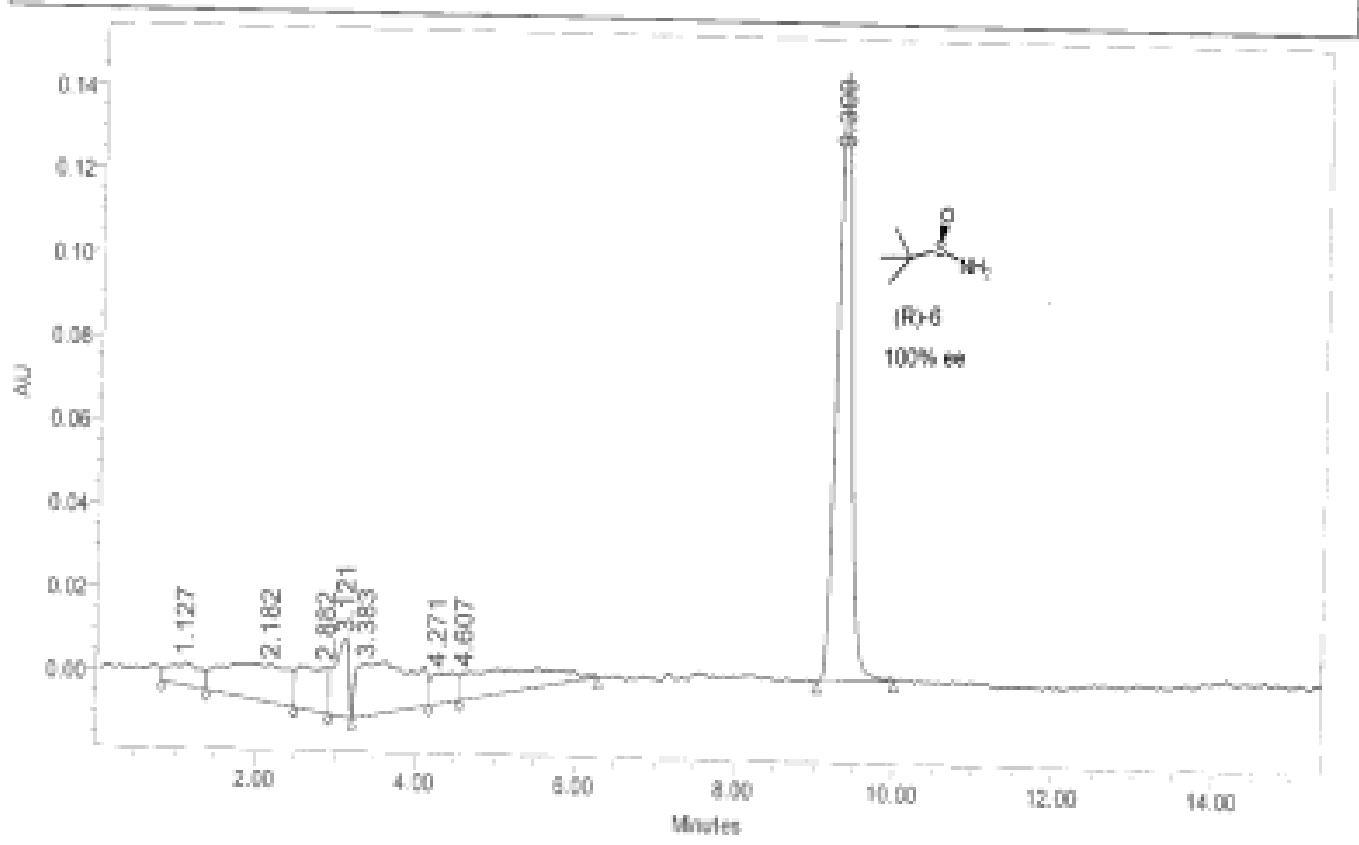

\begin{tabular}{|c|c|c|c|c|c|}
\hline & $\begin{array}{c}\text { RT } \\
\text { (min) }\end{array}$ & $\begin{array}{c}\text { Area } \\
\left(\mu V^{*} \mathrm{sec}\right)\end{array}$ & \%Area & $\begin{array}{c}\text { Height } \\
\left\langle M{ }^{2}\right.\end{array}$ & $\begin{array}{c}\% \\
\text { Height }\end{array}$ \\
\hline 1 & 1,127 & 144916 & 3.47 & 5621 & 259 \\
\hline 2 & 2182 & 502251 & 12.02 & 5036 & 4.16 \\
\hline & 2.882 & 200118 & 6.22 & 11094 & 5.07 \\
\hline & 3.121 & 226099 & 5.41 & 19561 & 8.91 \\
\hline & 3380 & 588877 & 14.04 & 12309 & 5.67 \\
\hline & 4.271 & $155 C 08$ & 3.73 & 7453 & 343 \\
\hline & 4.607 & 423305 & 10.13 & 8427 & 296 \\
\hline & 9.300 & 1880347 & 44.99 & 146025 & 67.22 \\
\hline
\end{tabular}




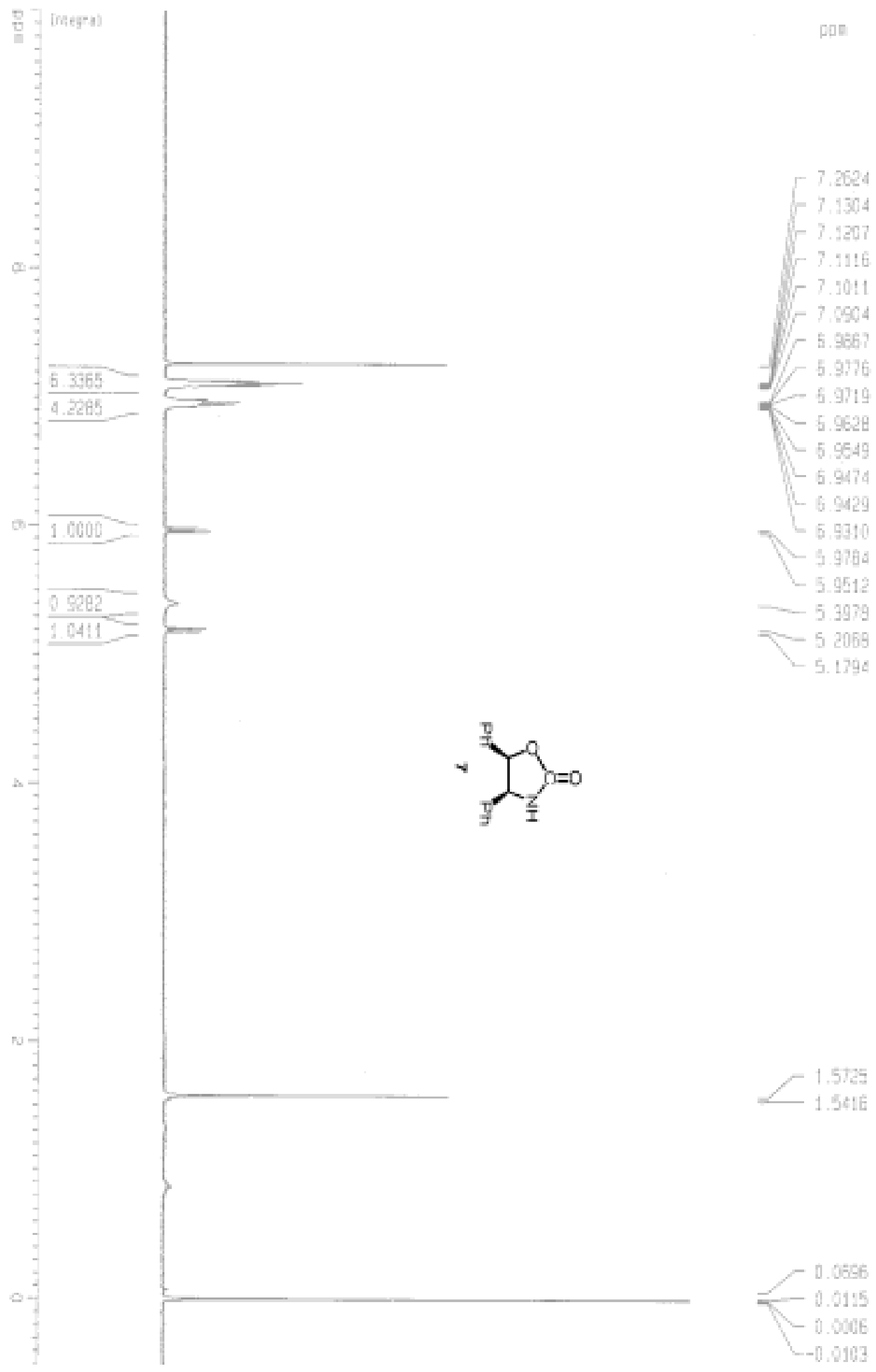




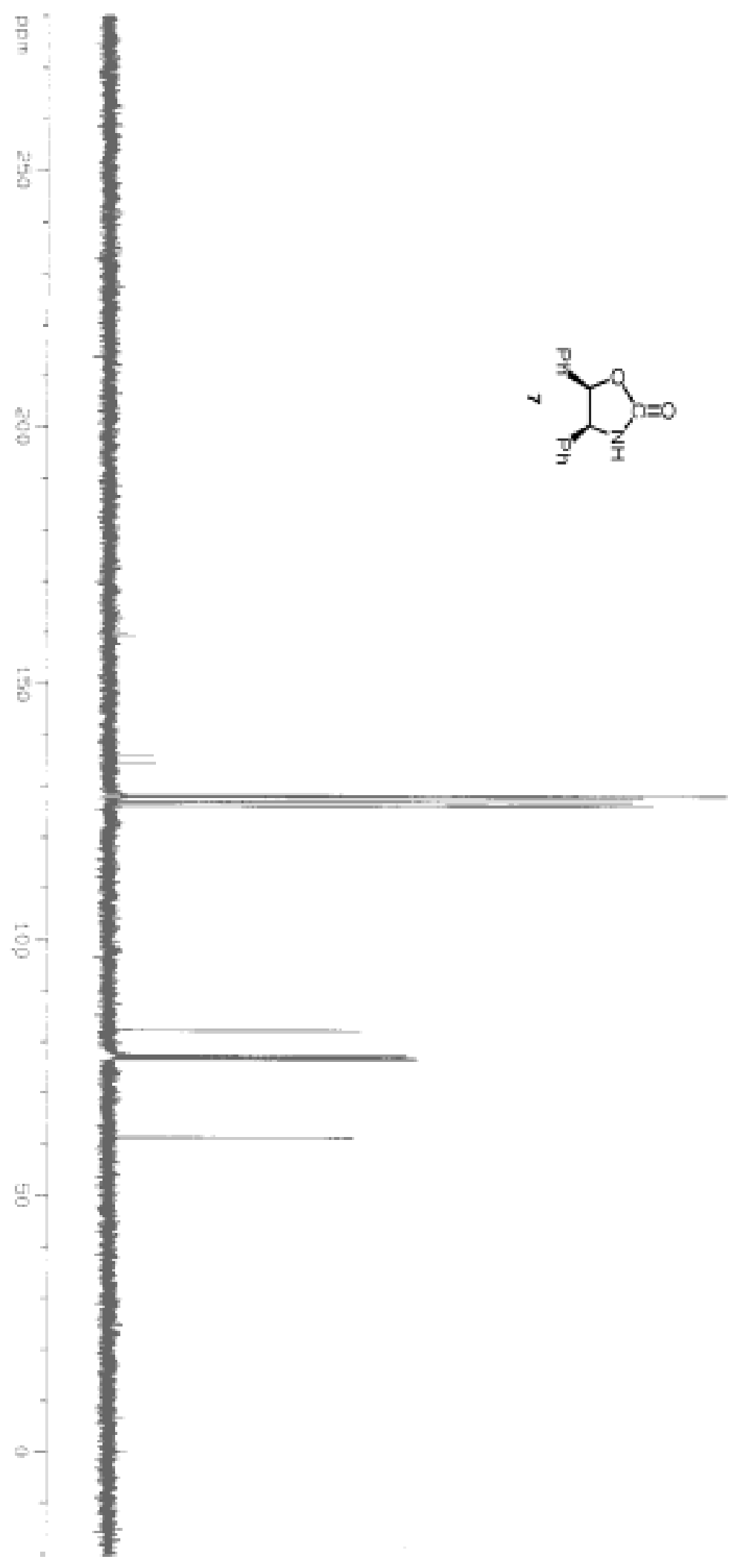

